Open Access

\title{
A high-resolution physical map integrating an anchored chromosome with the BAC physical maps of wheat chromosome $6 \mathrm{~B}$
}

Fuminori Kobayashi ${ }^{1}$, Jianzhong $\mathrm{Wu}^{1,2}{ }^{2}$, Hiroyuki Kanamori ${ }^{1}$, Tsuyoshi Tanaka ${ }^{3}$, Satoshi Katagiri ${ }^{2}$, Wataru Karasawa ${ }^{2}$, Satoko Kaneko ${ }^{4}$, Shota Watanabe ${ }^{4}$, Toyotaka Sakaguchi ${ }^{4}$, Yumiko Hanawa ${ }^{2}$, Hiroko Fujisawa ${ }^{2}$, Kanako Kurita ${ }^{1}$,

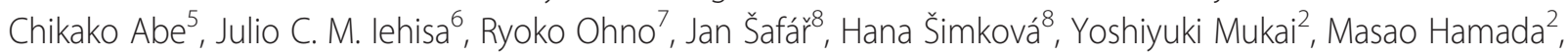
Mika Saito ${ }^{9}$, Goro Ishikawa ${ }^{9}$, Yuichi Katayose ${ }^{2}$, Takashi R. Endo ${ }^{4}$, Shigeo Takumi ${ }^{6}$, Toshiki Nakamura ${ }^{9}$, Kazuhiro Sato ${ }^{10}$, Yasunari Ogihara ${ }^{11}$, Katsuyuki Hayakawa ${ }^{5}$, Jaroslav Doležel ${ }^{8}$, Shuhei Nasuda ${ }^{4}$, Takashi Matsumoto $^{1}$ and Hirokazu Handa ${ }^{1 *}$

\begin{abstract}
Background: A complete genome sequence is an essential tool for the genetic improvement of wheat. Because the wheat genome is large, highly repetitive and complex due to its allohexaploid nature, the International Wheat Genome Sequencing Consortium (IWGSC) chose a strategy that involves constructing bacterial artificial chromosome (BAC)-based physical maps of individual chromosomes and performing BAC-by-BAC sequencing. Here, we report the construction of a physical map of chromosome $6 \mathrm{~B}$ with the goal of revealing the structural features of the third largest chromosome in wheat.

Results: We assembled 689 informative BAC contigs (hereafter reffered to as contigs) representing $91 \%$ of the entire physical length of wheat chromosome $6 \mathrm{~B}$. The contigs were integrated into a radiation hybrid (RH) map of chromosome 6B, with one linkage group consisting of 448 loci with 653 markers. The order and direction of 480 contigs, corresponding to $87 \%$ of the total length of $6 \mathrm{~B}$, were determined. We also characterized the contigs that contained a part of the nucleolus organizer region or centromere based on their positions on the RH map and the assembled BAC clone sequences. Analysis of the virtual gene order along $6 \mathrm{~B}$ using the information collected for the integrated map revealed the presence of several chromosomal rearrangements, indicating evolutionary events that occurred on chromosome 6B.
\end{abstract}

Conclusions: We constructed a reliable physical map of chromosome $6 \mathrm{~B}$, enabling us to analyze its genomic structure and evolutionary progression. More importantly, the physical map should provide a high-quality and map-based reference sequence that will serve as a resource for wheat chromosome 6B.

Keywords: Centromere, Chromosomal rearrangement, Chromosome 6B, DNA marker, Gene order, Nucleolus organizer region, BAC physical map, RH map, Synteny, Wheat

\footnotetext{
* Correspondence: hirokazu@affrc.go.jp

${ }^{1}$ Plant Genome Research Unit, National Institute of Agrobiological Sciences,

Tsukuba 305-8602, Japan

Full list of author information is available at the end of the article
} 


\section{Background}

Common wheat (Triticum aestivum L.) is an allohexaploid species with three distinct genomes, A, B and D, which have been defined by genome analysis [1]. The wheat genome consists of 21 chromosomes, and each of the three subgenomes contributes 7 chromosomes [2, 3]. The genome has a total length of $16.9 \mathrm{~Gb}$ [4], which is extremely large compared to the sequenced genomes of other grass species: rice (389 Mb; [5]), Brachypodium distachyon (272 Mb; [6]), sorghum (730 Mb; [7]), maize (2.3 Gb; [8]) and barley (5.1 Gb; [9]). Most of the wheat genome is occupied by various types of repetitive DNA sequences, which account for more than $80 \%$ of the entire genome [10]. Although wheat has been widely used in cytogenetics because of the high visibility of its chromosomes, complex features, such as its large size, high repeat content and polyploidy, have hampered molecular approaches to date, particularly whole-genome sequencing. However, recent technological advances have paved the way for genomic approaches that work for even complex genomes, such as wheat. For example, wholegenome sequences of the common wheat cultivar 'Chinese Spring' (CS), wild diploid wheat Triticum urartu (A genome progenitor of common wheat) and wild diploid goat grass Aegilops tauschii (D genome progenitor of common wheat) were analyzed using next-generation sequencing (NGS) technology [11-13].

The International Wheat Genome Sequencing Consortium (IWGSC) has been coordinating wheat genome sequencing to enhance knowledge of the structure and function of the bread wheat genome (http://www.wheat genome.org/) and to provide tools to facilitate the breeding of improved varieties. The consortium has adapted a strategy that relies on the purification of individual chromosome arms from wheat telosomic lines [2] by flow-cytometric sorting to reduce the sample complexity [14] and obtain chromosome-specific genomic data. Recently, the IWGSC presented a draft sequence of all 21 wheat chromosomes [15] in which the DNA of flowsorted fractions was sequenced by NGS. To obtain a reference genome sequence using BAC-by-BAC sequencing, physical mapping of the BAC contigs (hereafter reffered to as contigs) is currently underway for each of the 21 chromosomes [4]. To date, BAC-based physical maps have been developed for chromosomes $1 \mathrm{~A}, 1 \mathrm{~B}, 3 \mathrm{~B}$ and $6 \mathrm{~A}[16-21]$. Most recently, during the preparation of this paper, two additional physical maps for chromosome arms 3DS and 5DS were released [22, 23].

Our group is responsible for sequencing wheat chromosome 6B (http://komugigsp.dna.affrc.go.jp/index. html) under the framework of IWGSC. This chromosome is the third largest chromosome in common wheat, with an estimated molecular size of $914 \mathrm{Mb}$ : $415 \mathrm{Mb}$ representing the short arm (6BS) and $498 \mathrm{Mb}$ representing the long arm (6BL) [4]. As a first step, we conducted whole-chromosome shotgun sequencing using DNA that had been amplified from the flowsorted chromosome arms 6BS and 6BL with massively parallel 454 pyrosequencing [24]. The survey sequence data were highly informative for determining the genomic composition of chromosome 6B. Within a total of $508 \mathrm{Mb}$ of assembled sequence ( $56 \%$ of the size of $6 \mathrm{~B}$ ), 4798 gene loci were predicted, and more than $70 \%$ of the $6 \mathrm{~B}$ assembly consisted of repetitive sequences. Functional non-protein-coding RNAs, such as micro-RNA, transfer RNA and ribosomal RNA (rRNA), were also identified. In particular, the short arm (6BS) is characterized by the presence of secondary constriction, the nucleolus organizer region (NOR), which features a locus for the rRNA genes (rDNA locus) $[25,26]$. The NOR is designated as Nor-B2 [27]. We identified several contigs containing the rDNA locus, and they exhibited extremely high read depths, indicating that these contigs were part of the NOR on 6BS.

In this study, we established a BAC-based physical map of chromosome $6 \mathrm{~B}$ with the goal of developing a high-quality reference sequence for the wheat genome. To achieve this goal, two BAC libraries were constructed using arm-specific DNA samples. In addition, we used two recently developed genomic tools to physically map all of the contigs onto chromosome 6B. This process was performed using insertion site-based polymorphism (ISBP) markers (Kaneko et al. in preparation) that were designed from the junction sequences between transposable elements and their flanking sequences [28], and a radiation hybrid $(\mathrm{RH})$ mapping panel (Watanabe et al. in preparation) that was derived from a series of chromosome deletion lines generated by $\gamma$-ray irradiation [29]. Combining the above genomic resources with other wheat-specific data, we were able to develop a robust system to anchor and order contigs onto the highresolution $\mathrm{RH}$ map of chromosome 6B. The physical map not only allows us to understand key structural features and the evolutionary history of chromosome $6 \mathrm{~B}$ but will also enable the use of genome sequencing to create a high-quality map-based reference sequence in the near future.

\section{Results and discussion}

\section{Construction of 6B arm-specific BAC libraries}

Chromosome arms $6 \mathrm{BS}$ and $6 \mathrm{BL}$ were purified by flow-cytometric sorting from the double ditelosomic line 6B of CS [30], as described by Tanaka et al. [24]. The average purities in the sorted fractions were 85 and $92 \%$ for $6 \mathrm{BS}$ and $6 \mathrm{BL}$, respectively. High-molecular-weight DNA prepared from the purified arms was used to construct the BAC libraries TaaCsp6BShA and TaaCsp6BLhA, which were specific 
to $6 \mathrm{BS}$ and 6BL, respectively (http://olomouc.ueb.cas.cz/ dna-libraries/cereals). The libraries consisted of 57,600 and 76,032 BAC clones with average insert lengths of 132 and $130 \mathrm{~kb}$, representing 15.3 and 18 genome equivalents of the estimated size of $6 \mathrm{BS}$ and $6 \mathrm{BL}$, respectively (Table 1 ).

\section{Construction of a BAC-based physical map of wheat chromosome 6B}

Whole Genome Profiling (WGP ${ }^{\mathrm{Tw}}$ ) [31] was used to fingerprint a total of 41,472 and 49,920 BACs from 6BS and $6 \mathrm{BL}$, representing 13 times the total physical lengths of the arms, respectively (Additional file 1). After deconvolution for the assignment of high-quality reads, we made several modifications to the standard WGP procedure following Philippe et al. [32] to ensure a highly accurate physical map. We initially excluded BACs with less than $30 \%(<7$ tags $)$ or more than 2.5 times $(>51$ tags) the average number of tags per BAC from the analysis based on the average number of tags per BAC (20.6 and 21.8 for 6BS and 6BL, respectively) (Additional files 1 and 2). This filtering resulted in the availability of 28,828 and 38,953 BACs for assembly on $6 \mathrm{BS}$ and $6 \mathrm{BL}$, representing 9.2 and 10.2 times the total physical length, respectively (Additional file 3 ). Assembly of the fingerprints of 6BS and 6BL was performed using FingerPrinted Contigs (FPC) software [33]. A stepwise method was used to improve the quality of the contigs in the assembly, which was initially defined during the physical map construction of chromosome 3B [20] and then modified for the WGP fingerprints [32]. Using the 6B-specific parameters at final cut-off values of $1 \mathrm{e}^{-12}$ and $1 \mathrm{e}^{-11}$, which were determined based on the balance among the number of contigs, the number of Q-contigs and the coverage of the physical map, we constructed the first physical maps of 6BS and 6BL with 1069 and 514 contigs, respectively (Additional file 3 and Fig. 1; the URGI site of the wheat physical map viewer, https://urgi.versailles.inra.fr/ gb2/gbrowse/wheat_phys_pub/).

These physical maps still contained a large number of small contigs (2 BACs/contig), particularly on 6BS (Fig. 1d). However, the small contigs might be redundant because of their low-quality fingerprints containing genomic fragments that were already present in the large contigs. In addition, they had the potential to carry BAC clones derived from chromosomes other than $6 \mathrm{~B}$ due to

Table 1 Arm-specific BAC libraries on chromosome 6B

\begin{tabular}{lcc}
\hline & $6 \mathrm{BS}$ & $6 \mathrm{BL}$ \\
\hline Library code & TaaCsp6BShA & TaaCsp6BLhA \\
Number of clones & 57,600 & 76,032 \\
Average insert size & $132 \mathrm{~kb}$ & $130 \mathrm{~kb}$ \\
Coverage & $15.3 \mathrm{x}$ & $18.0 \mathrm{x}$ \\
Purity of the sorted fraction & $85 \%$ & $92 \%$ \\
\hline
\end{tabular}

DNA contamination during the chromosome-sorting process (the purity of the sorted fractions was approximately 85-92 \%; Table 1). To avoid any chromosomal redundancy and contamination, we eliminated 765 and 129 of these small and low-confidence contigs from the above physical map constructed primarily on the two arms of $6 \mathrm{BS}$ and $6 \mathrm{BL}$, respectively, based on the results of a homology search of the WGP tag sequences against the whole-genome survey sequence [15] and/or the depth of the BAC clones within each contig. Finally, we constructed the BAC-based physical map of chromosome arms $6 \mathrm{BS}$ and $6 \mathrm{BL}$, which contained 304 and 385 contigs, respectively (Table 2 ).

The above process greatly improved our BAC-based physical map. The first physical maps revealed estimated sizes of 492 and $495 \mathrm{Mb}$ that corresponded to $119 \%$ and $99 \%$ of the arm lengths, N50 values of 1503 and $2422 \mathrm{~kb}$ and L50 values of 87 and 65 contigs for 6BS and $6 \mathrm{BL}$, respectively (Additional file 4). However, the final maps showed remarkable increases in the N50 values (from 1503 to $2302 \mathrm{~kb}$ for $6 \mathrm{BS}$ and from 2422 to $2508 \mathrm{~kb}$ for 6BL) and a decrease in the L50 values (from 87 to 52 for $6 \mathrm{BS}$ and from 65 to 61 for $6 \mathrm{BL}$ ), although the total lengths for both arms decreased to 359 and $475 \mathrm{Mb}$, covering approximately 87 and $95 \%$ of the entire genomic region for $6 \mathrm{BS}$ and $6 \mathrm{BL}$, respectively (Additional file 4; Table 2). The reason for the lower coverage of $6 \mathrm{BS}$ remains unclear, but it is likely a consequence of the purity of the sorted chromosome arm DNA (Table 1), the number of WGP tags on each BAC (Additional file 2) or differences in the genomic structure between 6BS and 6BL (e.g., the Nor-B2 region with highly repetitive rRNA gene sequences).

Further overlap analysis of neighboring clones within each contig using the FPC MTP module allowed us to select 3076 and 4557 minimal tiling path (MTP) clones along the two arms, laying a foundation for the mapbased genomic sequencing of the entire chromosome $6 \mathrm{~B}$ (Table 2).

\section{Anchoring of chromosome 6B-specific markers to determine the physical location of the BAC contigs}

To obtain a pseudomolecule sequence, which is the final goal of IWGSC, it is essential to map the contigs onto their specific genomic regions on wheat chromosome $6 \mathrm{~B}$. For that purpose, a set of anchors is needed. As shown in Table 3, we first searched through the available marker information in public databases, such as the "GrainGenes CMap" (http://wheat.pw.usda.gov/cmap/) and the "National BioResource Project (NBRP)-Wheat, Japan" (http://www.shigen.nig.ac.jp/wheat/komugi/), and 208 SSR and RFLP markers (already converted to STS markers) were found to be potentially useful for chromosome 6B. Second, we collected a resource of 751 DNA 

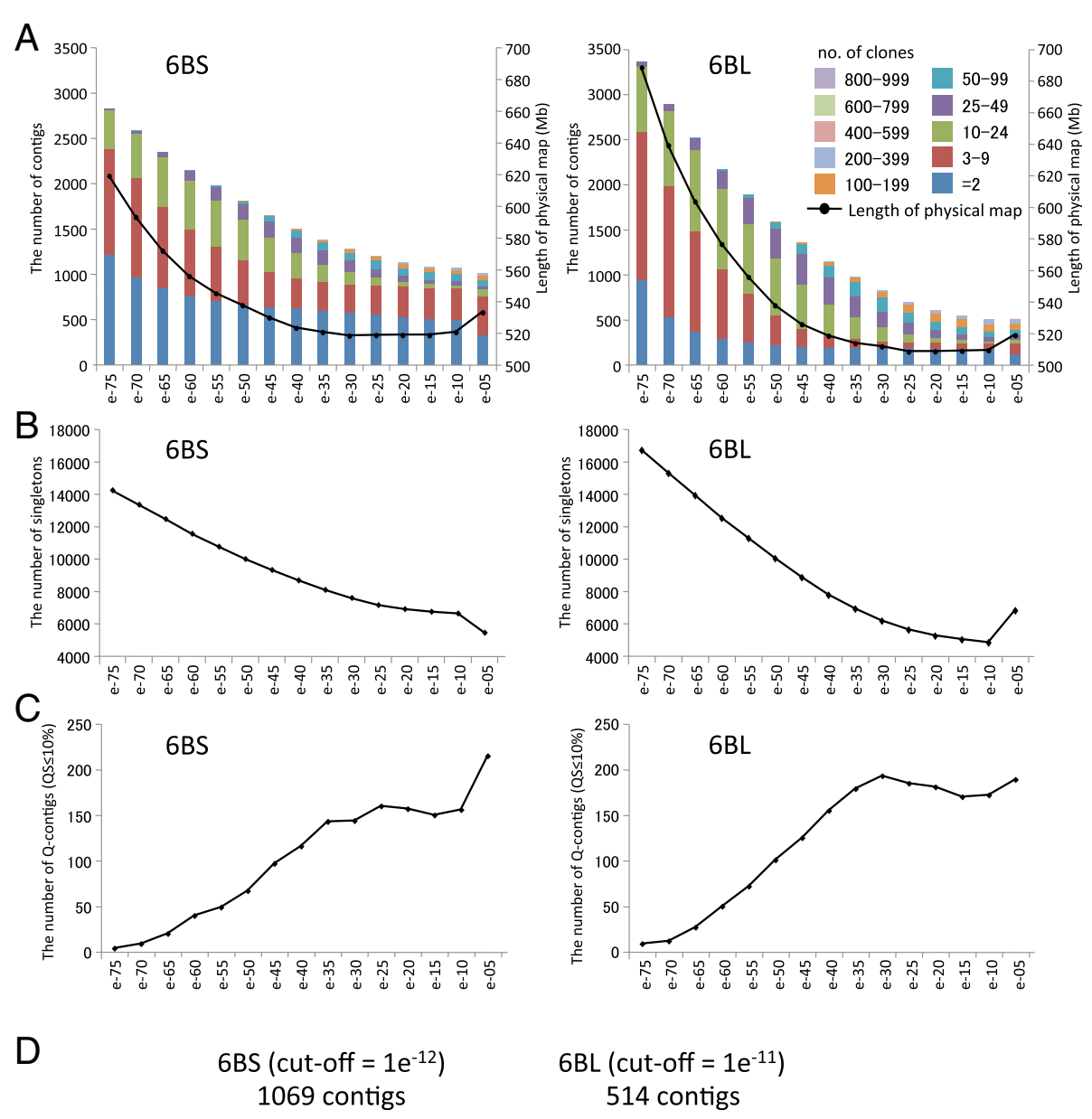
6BL (cut-off $=1 \mathrm{e}^{-11}$ ) 514 contigs
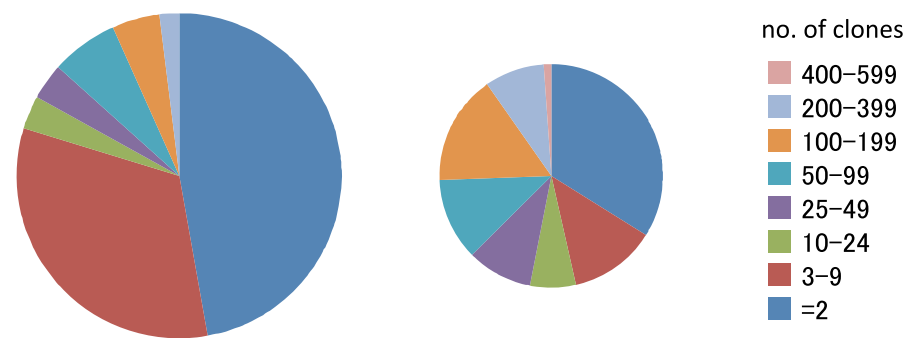

Fig. 1 Comparison of the contig assemblies between cut-offs of $1 \mathrm{e}^{-75}$ and $1 \mathrm{e}^{-05}$ in the chromosome arms of $6 \mathrm{BS}$ and 6BL. a The number of contigs and their cumulative sizes at each cutoff value. The colors in the bars represent the number of clones comprising one BAC contig. $\mathbf{b}$ Total number of singleton clones in the physical map at each cut-off value. c Total number of Q-contigs containing $\leq 10 \%$ questionable clones in the physical map at each cut-off value. $\mathbf{d}$ Total number of contigs in the physical maps of $6 \mathrm{BS}$ and $6 \mathrm{BL}$ with an optimal cut-off value. Eight colors in circles represent the number of clones comprising one BAC contig. The sizes of the circles indicate the relative total number of contigs in the physical map of 6BS and 6BL

markers from the PCR-based Landmark Unique Gene (PLUG) Database (http://plug.dna.affrc.go.jp/). These markers are known EST-PCR markers that were developed by taking advantage of the orthologous gene conservation between rice and wheat $[34,35]$. To develop new markers, we designed 390 and 1829 PCR primer pairs from the genic sequences by using the barley GenomeZipper (chromosome 6H) [36] and the syntenic relationships of rice (chromosome 2), B. distachyon (chromosome 3) and sorghum (chromosome 4), respectively. Finally, using the survey sequences of wheat chromosome 6B [15], we prepared an additional 2000 ISBP PCR primer pairs (1000 for each chromosome arm) from the junction sequences between transposable elements and their flanking sequences ([28], Kaneko et al. in preparation).

After validating the quality and effectiveness of the above 5178 DNA markers by PCR, we found that up to 2584 primer pairs (1191 for 6BS and 1393 for 6BL) could amplify a single sharp band from the DNA samples of flow-sorted chromosome $6 \mathrm{~B}$ arms or pooled 
Table 2 Physical maps of chromosome arms 6BS and 6BL

\begin{tabular}{lcc}
\hline & $6 \mathrm{BS}$ & \multicolumn{2}{c}{$6 \mathrm{BL}$} \\
\hline Number of BACs in the contigs & 20,225 & 33,830 \\
Number of contigs & 304 & 385 \\
Estimated size (chromosome & $359 \mathrm{Mb}(87 \%)$ & $475 \mathrm{Mb}(95 \%)$ \\
coverage $^{\mathrm{a}}$ ) & & \\
Contigs N50 (kb) & 2302 & 2508 \\
Contig L50 & 52 & 61 \\
Number of MTP BACs & 3076 & 4557 \\
\hline
\end{tabular}

${ }^{a}$ Coverage was calculated based on the estimated size of each arm: $415 \mathrm{Mb}$ for $6 \mathrm{BS}$ and $498 \mathrm{Mb}$ for $6 \mathrm{BL}$

arm-specific BAC libraries. These primer pairs represent good markers of the chromosomal locations of the contigs (Table 3). As shown in Fig. 2, using largescale PCR-screening of two arm-specific BAC libraries, we successfully localized the above 2584 anchor markers onto individual BAC clones among a total of 449 contigs (199 on $6 \mathrm{BS}$ and 250 on 6BL, 5.8 markers per contig). The ISBP markers provided more effective anchoring of the contigs compared to the genic markers (89 \% versus 77-80 \%), supporting previous results ([20]; Kaneko et al. in preparation). In addition, 271 markers were specific to wheat chromosome 6B and were polymorphic between the two wheat varieties CS and 'Mironovskaya 808' (M808), which were the parents used to generate the mapping population of recombinant inbred lines (RILs) [37] that was used for genetic mapping of contigs.

To anchor the contigs without any of 2584 6B-specific anchor marker hits, we developed a point-by-point supplemental set of 284 novel 6B-specific markers (92 converted from the markers specific to wheat group-6 chromosomes using the survey sequence data [15] and 192 from the genomic sequences of the MTP BAC clones in the corresponding contigs). Finally, the total number of contigs anchored by the chromosome 6B markers was increased to 475, including 207 contigs anchored by 1324 markers on 6BS and 268 contigs anchored by 1544 markers on 6BL (Table 4).

Table 3 Molecular markers used for the physical mapping

\begin{tabular}{lccc}
\hline Marker category & Developed & $\begin{array}{l}\text { Anchored to BAC } \\
\text { contig } \\
\text { (success rate) }\end{array}$ & $\begin{array}{l}\text { On } \\
\text { integrated } \\
\text { physical } \\
\text { map }\end{array}$ \\
\hline $\begin{array}{l}\text { Genic markers } \\
\text { a }\end{array}$ & 2970 & $1164(39.2 \%)$ & 1159 \\
$\begin{array}{l}\text { SSR and STS-RFLP } \\
\text { markers }\end{array}$ & 208 & $127(61.1 \%)$ & 127 \\
ISBP marker & 2000 & $1293(64.7 \%)$ & 1290 \\
Total & 5178 & $2584(50.0 \%)$ & 2576 \\
\hline
\end{tabular}

${ }^{a}$ Genic markers include the PLUG marker, GenomeZipper-based markers and orthologous sequence markers

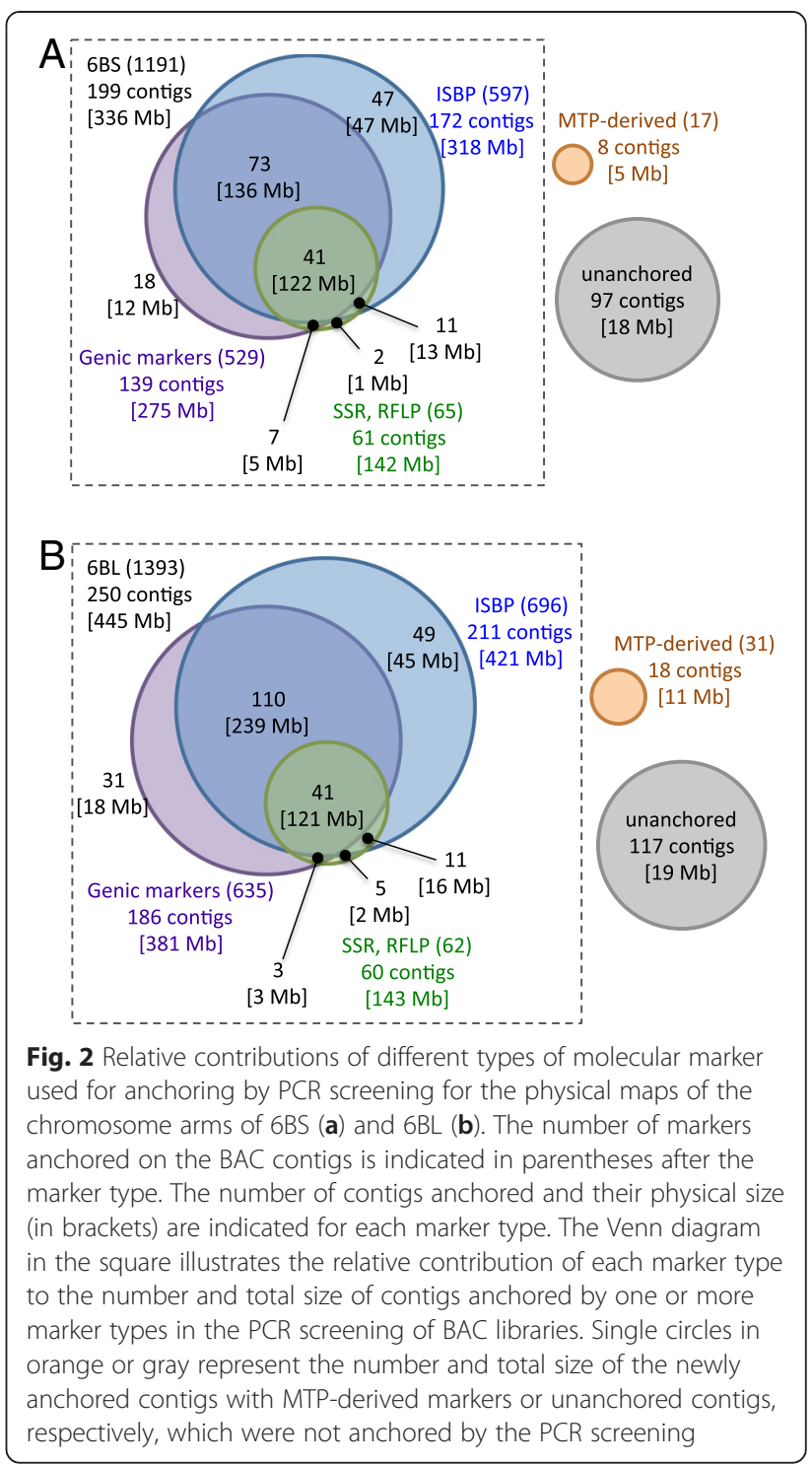

First, we attempted the genetic mapping of contigs on chromosome 6B. The use of 226 genetic markers located on the genetic map generated with the RIL population mentioned above led to the chromosomal assignment of 118 contigs (58 on 6BS and 60 on 6BL), encompassing a genomic region of $287 \mathrm{Mb}$, on the two arms, which represents $31 \%$ of chromosome $6 \mathrm{~B}$ (data not shown). However, genetic markers alone were not sufficient for the physical mapping of all contigs that were assembled for wheat chromosome $6 \mathrm{~B}$. To overcome this problem, we used RH mapping in chromosome deletion lines that were produced by $\gamma$-ray irradiation to determine the chromosomal locations and orders of all of the anchor DNA markers for the $6 \mathrm{~B}$ contigs. This strategy was used because $\mathrm{RH}$ mapping provides results that are independent of polymorphisms. Thus, it is a powerful tool for the high-resolution mapping of wheat chromosomes [29, 38]. 
Table 4 Characteristics of the BAC contigs in the physical map of chromosome 6B

\begin{tabular}{lccc}
\hline & 6BS (size, coverage $\left.{ }^{\mathrm{a}}\right)$ & 6BL (size, coverage $\left.{ }^{\mathrm{a}}\right)$ & 6B (size, coverage $\left.{ }^{\mathrm{a}}\right)$ \\
\hline BAC contigs on the RH map & $201(340.2 \mathrm{Mb}, 82.0 \%)$ & $261(454.5 \mathrm{Mb}, 91.3 \%)$ & $462(794.8 \mathrm{Mb}, 87.0 \%)$ \\
BAC contigs not on the RH map but anchored with markers & $6(1.05 \mathrm{Mb}, 0.25 \%)$ & $7(1.12 \mathrm{Mb}, 0.22 \%)$ & $13(2.17 \mathrm{Mb}, 0.24 \%)$ \\
BAC contigs not anchored with markers & $97(17.8 \mathrm{Mb}, 4.3 \%)$ & $117(19.0 \mathrm{Mb}, 3.8 \%)$ & $214(36.8 \mathrm{Mb}, 4.0 \%)$ \\
\hline
\end{tabular}

${ }^{\mathrm{a}}$ Coverage was calculated based on the estimated size of each arm and the entire chromosome: $415 \mathrm{Mb}$ for $6 \mathrm{BS} ; 498 \mathrm{Mb}$ for $6 \mathrm{BL}$; and $914 \mathrm{Mb}$ for $6 \mathrm{~B}$

Using the RH panel of 355 lines and 21 chromosome 6B deletion lines made by Endo and Gill [39], we established an $\mathrm{RH}$ map with one linkage group consisting of 653 markers (448 loci), in which a total of 1277 obligate breaks were identified with an estimated resolution of approximately $622 \mathrm{~kb} / \mathrm{break}$ (Table 5; Fig. 3b). The total length of the RH map was $1560.7 \mathrm{cR}$, indicating an average space of $3.5 \mathrm{cR} /$ loci and an average distance of $509 \mathrm{~kb} / \mathrm{cR}$ (the physical length used herein was estimated from the cumulative size of the contigs). Based on the RH mapping results, $462 \mathrm{FPC}$-assembled contigs were mapped. However, 18 of these mapped contigs (4 on 6BS and 14 on 6BL) were judged to be chimeric because the markers anchored to the contig were located on distant, unrelated positions of the RH map. Therefore, we split each of these 18 contigs into two distinct contigs based on the FPC data and the anchored marker positions.
Finally, we determined the locations and/or directions of 480 contigs on wheat chromosome $6 \mathrm{~B}$, including the contigs that mapped to the same locus (Additional file $5)$. These contigs revealed a total physical length of 794.8 Mb (340.2 and 454.5 Mb, respectively, on 6BS and $6 \mathrm{BL}$ ), representing approximately $87 \%$ of chromosome 6B (Table 4). Although 227 contigs, one third of the total contigs, still remained unmapped, most of these contigs were assembled using a small number of BAC clones with a total physical length of $39.0 \mathrm{Mb}$ and an average size of $172 \mathrm{~kb}$ per contig (maximum $=386 \mathrm{~kb}$, minimum $=81 \mathrm{~kb}$ ), so they cover only $4.2 \%$ of chromosome 6B.

Our RH mapping could offer more refined deletion bins for chromosome $6 \mathrm{~B}$ than were previously available [40]. The 18 breakpoints in the $6 \mathrm{~B}$ deletion lines and 653 markers defined 20 chromosomal blocks (7 on 6BS

Table 5 Integrated physical map and map resolution

\begin{tabular}{|c|c|c|c|c|c|c|c|}
\hline Deletion bin & Markers & Loci & Length $(\mathrm{CR})$ & Mapped contigs & Mapped contig size (Mb) & Obligate breaks $^{a}$ & Map resolution (Mb) \\
\hline $6 \mathrm{BS7}$ & 91 & 64 & 174.6 & 64 & 84.3 & 180 & 0.47 \\
\hline $6 \mathrm{BS9}$ & 34 & 22 & 83.8 & 19 & 26.8 & 57 & 0.47 \\
\hline $6 \mathrm{BS} 8$ & 25 & 15 & 33.1 & 17 & 33.0 & 29 & 1.14 \\
\hline $6 \mathrm{BS} 5$ & 18 & 13 & 35.8 & 15 & 26.4 & 32 & 0.83 \\
\hline $6 \mathrm{BS} 4$ & 54 & 43 & 145.4 & 39 & 76.9 & 115 & 0.67 \\
\hline $6 \mathrm{BS1}$ & 35 & 30 & 209.1 & 29 & 61.5 & 134 & 0.46 \\
\hline C-6BS1 & 34 & 21 & 102.7 & 22 & 31.5 & 58 & 0.54 \\
\hline C-6BL12 & 13 & 10 & 42.2 & 10 & 7.6 & 19 & 0.40 \\
\hline 6BL12 & 15 & 11 & 50.8 & 11 & 17.1 & 27 & 0.63 \\
\hline 6BL13 & 39 & 33 & 154.3 & 34 & 60.3 & 103 & 0.59 \\
\hline 6BL10 & 9 & 8 & 25.4 & 6 & 7.3 & 16 & 0.46 \\
\hline 6BL6 & 18 & 16 & 48.8 & 15 & 28.6 & 36 & 0.79 \\
\hline 6BL11 & 17 & 9 & 41.7 & 14 & 18.1 & 27 & 0.67 \\
\hline $6 \mathrm{BL} 3$ & 9 & 7 & 21.1 & 8 & 21.4 & 13 & 1.65 \\
\hline $6 \mathrm{BL4}$ & 11 & 11 & 34.5 & 9 & 10.5 & 25 & 0.42 \\
\hline $6 \mathrm{BL} 5$ & 69 & 50 & 155.2 & 51 & 87.6 & 128 & 0.68 \\
\hline 6BL8 & 3 & 2 & 5.8 & 1 & 3.6 & 2 & 1.80 \\
\hline 6BL9 & 29 & 19 & 43.9 & 25 & 35.0 & 36 & 0.97 \\
\hline 6BL1 & 2 & 2 & 3.2 & 1 & 1.7 & 2 & 0.84 \\
\hline $6 \mathrm{BL} 14$ & 128 & 62 & 149.3 & 90 & 155.7 & 144 & 1.08 \\
\hline $6 B$ & 653 & 448 & 1560.7 & 480 & 794.8 & 1277 & 0.62 \\
\hline
\end{tabular}

${ }^{\mathrm{a}}$ The number of obligate breaks in each deletion bin was calculated by using the map region between the distal and proximal markers 


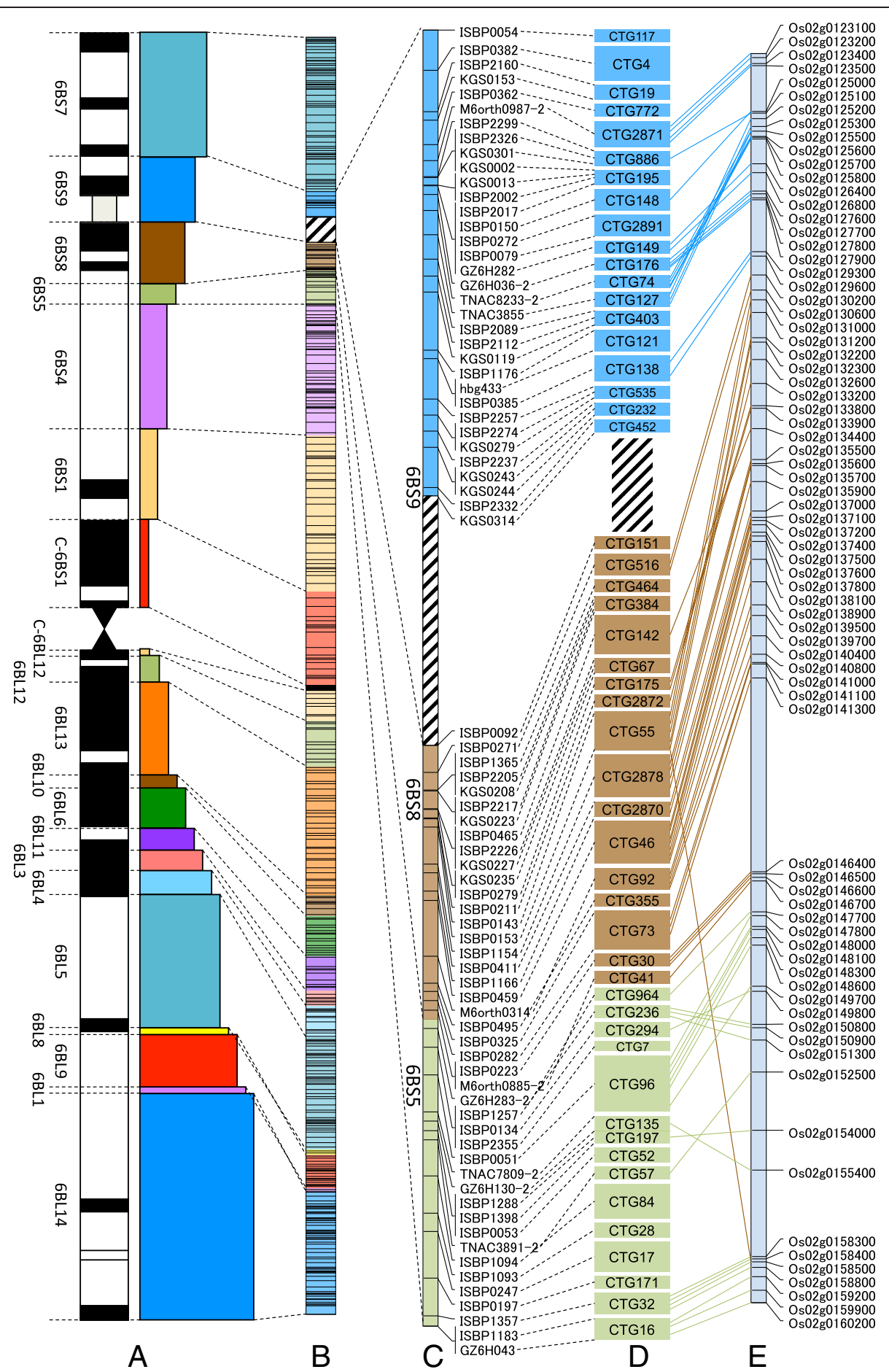

Fig. $3 \mathrm{RH}$ map of chromosome $6 \mathrm{~B}$ and contig order in three deletion bins of $6 \mathrm{BS}$. a Deletion bin map of chromosome 6B. Twenty deletion bins are illustrated by colored boxes. b RH map of chromosome 6B. The map is divided into segments corresponding to deletion bins with the same colors. The striped segment on 6BS represents the putative nucleolus organizer region (Nor-B2). The segment colored in black represents a boundary between 6BS and 6BL. c RH map at deletion bins 6BS9, 6BS8 and 6BS5. The marker name is indicated to the right of the chromosome. d BAC contigs assigned to the RH map. The name of the BAC contigs is displayed in colored boxes, the size of which reflects the relative contig size. The colors of the boxes correspond to those of the deletion bins. e Corresponding region of rice chromosome 2. Each dashed line represents the relationship between a genic marker located in a wheat BAC contig and the orthologous rice gene 
and 13 on 6BL). These 20 blocks were designated as improved deletion bins (Fig. 3a). Each deletion bin was denoted by the name of the deletion line and the breakpoint at the proximal end of the bin (Fig. 3a). Based on the number of obligate breaks and the bin size (calculated from the cumulative size of the contigs), the resolution of all 20 deletion bins was estimated to range from 0.4 to $1.8 \mathrm{Mb} /$ break (Table 5). Five bins (6BS8, 6BL3, 6BL8, 6BL9 and 6BL14) showed a relatively low resolution with $>900 \mathrm{~kb} / \mathrm{break}$, which may have occurred for the following reasons: 1 ) the large cumulative size of the contigs with a limited number of obligate breaks (e.g., 6BS8, 6BL3 and 6BL9); 2) one locus mapped by a large number of markers (e.g., 6BL14); and 3) a bin mapped with only one large contig (e.g., 6BL8). Deletion bins that mapped to regions close to the centromere a remarkably high resolution. For example, bin C-6BL12 had the highest resolution, $0.40 \mathrm{Mb} /$ break (Table 5). This result shows that RH mapping is useful for the chromosomal assignment of contigs, even within the centromeric region, where recombination events are extremely rare.

Notably, the largest space between markers on the $\mathrm{RH}$ map was located between KGS0314 (6BS9) and ISBP0092 (6BS8), which were separated by $29.2 \mathrm{cR}$ (Fig. 3c). Clearly, this chromosomal site was associated with the chromosome breakpoint of deletion line 6BS-9, which was located near Nor-B2 [39]. These results suggest that this space over $29.2 \mathrm{cR}$ corresponds to the NOR region (see the following section).

Although 350 of the 480 contigs (152 on 6BS and 198 on $6 \mathrm{BL}$ ) were specifically positioned in order along chromosome 6B (Additional file 5), 130 contigs were localized within a total of 47 loci (16 on 6BS and 31 on $6 \mathrm{BL}$ ), which indicated that multiple contigs resided within the same locus. For example, 4 of the 13 loci within the bin of 6 BL14 carried 5 to 7 contigs in which the relative order was unknown at this stage. However, 80 of these 130 contigs ( 21 on $6 \mathrm{BS}$ and 59 on $6 \mathrm{BL}$ ) were anchored by genic markers with orders that were estimated from the syntenic relationships of rice, $B$. distachyon and sorghum. These efforts led to the successful determination of the physical locations of a total of 430 contigs, representing a total length of $761.6 \mathrm{Mb}$ and covering approximately $83 \%$ of chromosome $6 \mathrm{~B}$ (Additional file 5).

In the present study, we developed an $\mathrm{RH}$ map for chromosome $6 \mathrm{~B}$ and determined the order of the contigs. Our study clearly demonstrates the appropriateness and effectiveness of the RH mapping approach for ordering contigs on wheat chromosome compared to typical strategies, such as genetic mapping and deletion bin mapping. The $\mathrm{RH}$ mapping method has important advantages. It produces uniform chromosomal breaks, allows for direct localization and does not require marker polymorphisms. Thus, it allows for the determination of the chromosomal locations and order of contigs with high resolution and accuracy.

$\mathrm{RH}$ mapping was able to define the order of 2860 markers based on contig order (Additional file 5). The marker order on the physical $\mathrm{RH}$ map provides more detail and a higher resolution compared to previous deletion bin mapping of chromosome 6B [35, 40-43]. Although the marker density of the $6 \mathrm{~B} \mathrm{RH}$ map (3.6 markers $/ \mathrm{Mb}$ ) is lower than those of other physical maps (10.1 and 11 markers/Mb on $1 \mathrm{BS}$ and 1BL, respectively; $[18,19])$, all of our markers are PCR markers, which will be valuable for future map-based gene cloning and marker-assisted selection in breeding compared to the array-based markers used in the previous studies.

\section{Fine mapping of the Nor-B2 region on the short arm of chromosome 6B}

The integration of FPC-assembled BAC contigs with a high-resolution $\mathrm{RH}$ map of chromosome $6 \mathrm{~B}$ allowed us to characterize several features that are specific to the genomic composition and structure of chromosome 6B. In common wheat, the majority of the highly repeated rRNA genes for 18S, 5.8S and 25S RNAs (rDNA unit) are located at the NOR loci on chromosome arms 1BS and 6BS, as well as at minor sites on chromosome arms $1 \mathrm{AS}$ and 5DS, which have been designated Nor-B1, Nor-B2, Nor-A1 and Nor-D3, respectively [27]. Nor-B2 is recognized as the secondary constriction on satellite chromosome $6 \mathrm{~B}$, which indicates that genes in Nor-B2 are transcriptionally active [26].

Our RH mapping defined a position for Nor-B2 on chromosome 6BS (Fig. 3b, c). To determine the physical position of Nor-B2 in detail, we developed one DNA marker using the intergenic spacer sequences (IGSs) between the 18S and 25S rRNA genes [44]. Screening of our BAC libraries with the IGS marker revealed that two contigs, CTG151 (2.6 Mb) and CTG142 (3.7 Mb), located in the deletion bin of 6BS8, putatively contained the rRNA genes (Fig. 3d). Contig CTG151 was mapped onto a genomic region immediately downstream of the estimated Nor-B2 site using one ISBP marker, ISBP0092, and contig CTG142 had a chromosomal position that was $7.5 \mathrm{cR}$ downstream of that marker on the RH map. Although three other contigs, CTG516 (1.9 Mb), CTG464 (0.4 Mb) and CTG384 (0.8 Mb), were located between CTG151 and CTG142 (Fig. 3d), no MTP clones were detected in these contigs by PCR screening using the IGS marker. However, BLAST analysis of genomic sequences of MTP BAC clones (unpublished data) clearly confirmed the presence of an rDNA unit within the BAC contigs of not only CTG151 and CTG142 but also CTG516 (Fig. 4). 


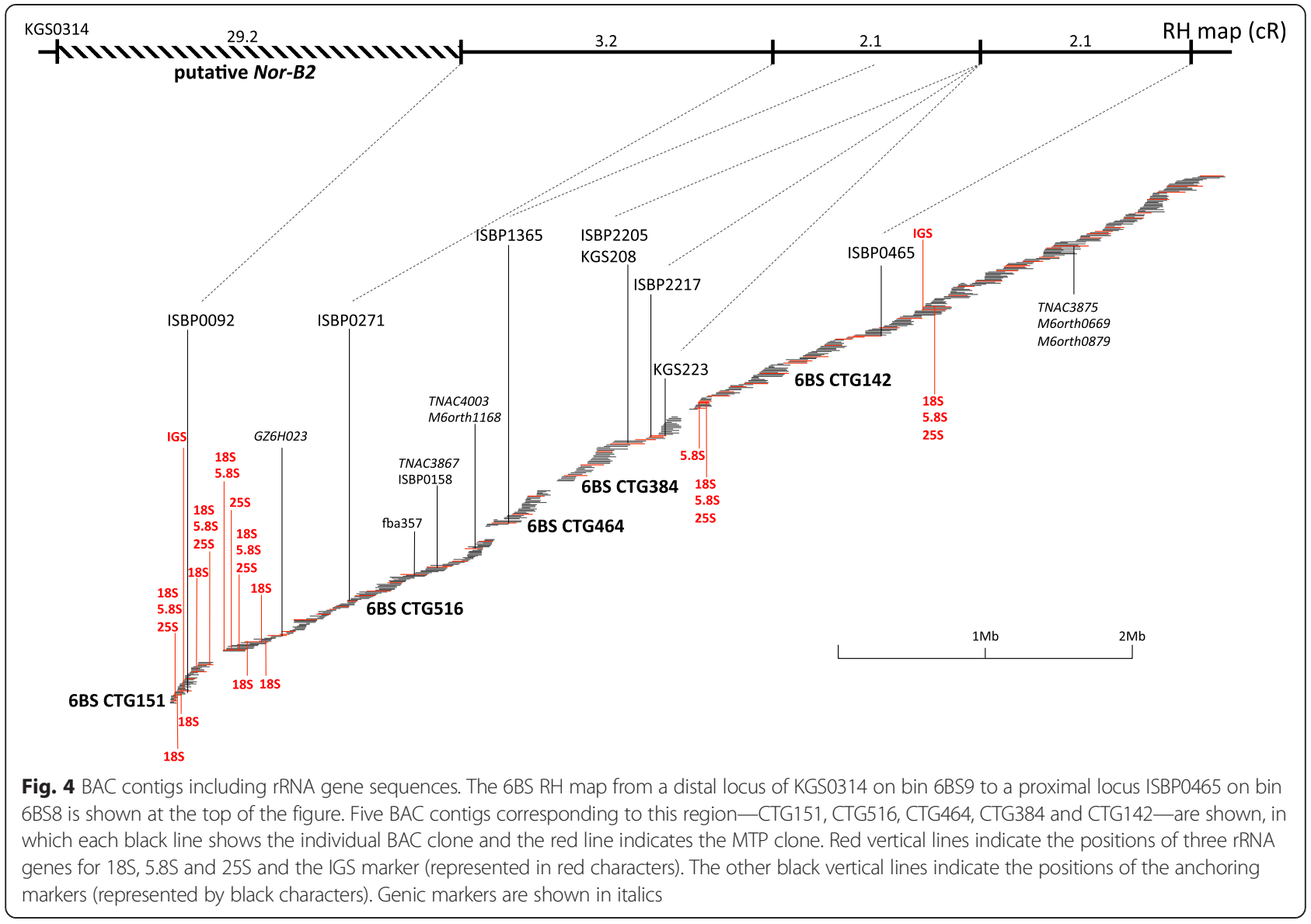

Previous research has identified approximately 5500 rRNA genes on chromosome 6B [45]. Considering that the length of each copy (rDNA unit and adjacent IGS) is approximately $9 \mathrm{~kb}$ [46], the complete region encompassing the Nor-B2 locus could be estimated as approximately $16.5 \mathrm{Mb}$. Although the five BAC contigs showed a total physical length of $9.4 \mathrm{Mb}$, the copy numbers of the rRNA genes detected within them were extremely limited (Fig. 4). Therefore, the current BAC-based physical map partially covers the border region (proximal to the centromere) of the Nor-B2 locus, and it does not include the core rDNA array region of Nor-B2. Furthermore, the physically mapped BAC contigs in the genomic region on the other side (proximal to telomere) of the Nor-B2 locus do not contain the rRNA genes.

Interestingly, all of the rRNA genes discovered on the above contigs that mapped near the Nor-B2 locus appeared to be interrupted by other types of genomic sequences, including transposons and other genes. This is in striking contrast to the NOR region in rice. In rice (Oryza sativa L. ssp. japonica), the rRNA genes are distributed in a uniform array throughout the NOR, encompassing a region of approximately $3.5 \mathrm{Mb}$ on chromosome 9 (Additional file 6) [47, 48]. Moreover, the rDNA units have been found to exist as clusters in tandem arrays, even within the border region of the NOR, demonstrating a clear boundary between the rDNA repeat and its flanking region [49]. In the region flanking the rDNAs on rice chromosome 9, Ty3/gypsy retrotransposons accumulates at a density twice as high as that of the entire rice genome $[5,49]$. Regarding chromosome $6 \mathrm{~B}$, two ISBP markers, ISBP0092 and ISBP0465, were designed based on the junction sequences, including the gypsy-type retrotransposon (Additional file 6), and these mapped close to the positions of the rRNA genes on CTG151 and CTG142, respectively (Fig. 4). The other five ISBP markers mapped to the same region (Fig. 4), including other types of repeat sequences, such as DNA transposons (CACTA and MITE) and unknown repeat sequences (Additional file 6). We previously found that the DNA transposon diffusion is involved in the propagation of micro RNA genes and specific transfer RNA genes in wheat chromosome $6 \mathrm{~B}$ based on the analysis of $6 \mathrm{~B}$ survey sequences [24]. The ambiguity found in the border region of Nor-B2 might be another example of specific transposons containing rRNA genes becoming 


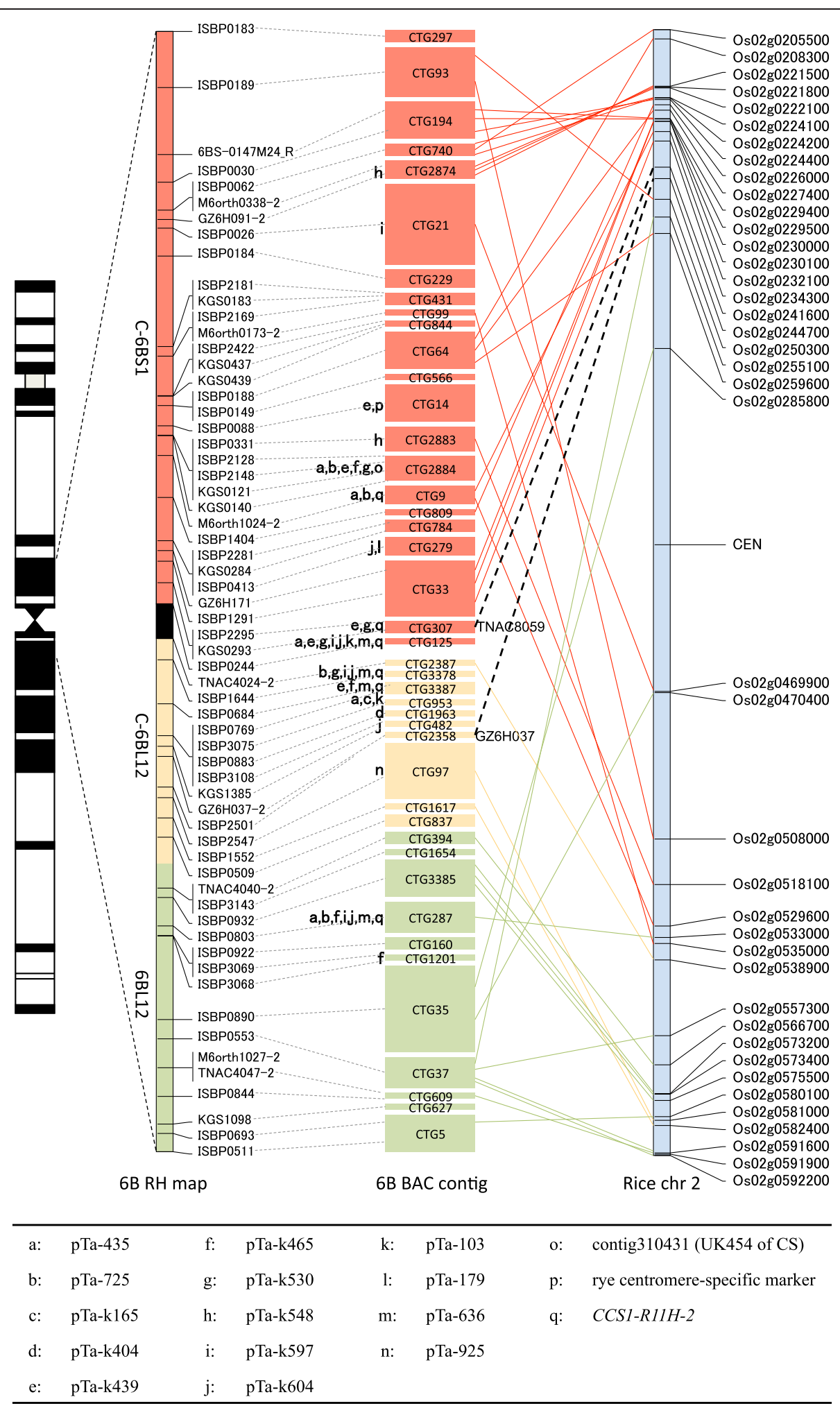

Fig. 5 (See legend on next page.) 
(See figure on previous page.)

Fig. 5 BAC contigs located in the centromeric region of chromosome 6B. The proximal three bins on chromosome 6B, C-6BS-1, C-6BL-12 and 6BL12, are represented in colored segments of the $\mathrm{RH}$ map, corresponding to the colors of each bin in Fig. 4 a. The black segment represents the boundary between $6 \mathrm{BS}$ and $6 \mathrm{BL}$. The marker name is indicated to the right of the map. BAC contigs assigned to the RH map are represented by colored boxes, the sizes of which reflect the relative contig sizes. The names of 17 centromeric sequences used as queries for the BLAST search are listed in the table at the bottom of the figure, and each sequence is represented by a letter from ' $a$ ' to ' $q$ '. These letters, shown on the right of the BAC contigs, denote the presence of the corresponding centromeric sequences in those contigs. The corresponding region of rice chromosome 2 is aligned on the right side of the figure. Each colored line represents the relationship between a genic marker located in a wheat BAC contig and the orthologous rice gene. Black dashed lines indicate the relationships between two wheat centromeric genic markers, TNAC8059 on CTG307 (C-6BS1) and GZ6H037 on CTG2358 (C-6BL12), and their orthologous genes in rice

diffused around the border region of Nor-B2, resulting in insertions of the rRNA genes into the genomic sequence outside of the rDNA array. We identified three gene loci (GZ6H023 and TNAC3867 on CTG516, TNAC3875 on CTG142) that displayed colinearity between wheat and rice (Figs. 3e and 4), and other mapped genes on CTG516 were also found to have conserved gene order relative to rice chromosome 2 . These results support the concept that the border region of Nor-B2 originally possessed a structure similar to that of rice; however, the occurrence of genomic rearrangements due to the transposition of various repeat sequences has resulted in the present ambiguity at the proximal border of Nor-B2. This feature may reflect general characteristics of the wheat genome, which contains highly repetitive sequences. A detailed evaluation of the structure of Nor-B2 and its relationship to the repetitive sequences will be presented in future sequencing studies.

\section{Fine mapping of the centromere on wheat chromosome $6 \mathrm{~B}$} In multicellular eukaryotes, the chromosomal region known as the centromere plays an important role in both mitotic and meiotic nuclear divisions. Centromeres in wheat are associated with highly repetitive Ty3/gypsy elements. Moreover, the regions surrounding centromeres (pericentomeres) are highly heterochromatic. In the present study, a total of 43 contigs were mapped within a genomic region represented by deletion bins of C-6BS1, C-6BL12 and 6BL12, where the centromere of wheat chromosome 6B should be located (Fig. 5). A number of centromere-specific sequences have been reported for wheat and its relatives, including 15 Ty3/gypsy-type centromeric retrotransposons in wheat $(C R W)$ [50], one centromeric satellite-like repeat (contig310431) in wheat [51], one Ty3/gypsy retrotransposon (CCS1-R11H-2) in Ae. tauschii [52], and one DNA marker sequence (KF719092) in rye. Using the 18 centromere-specific sequences described above, we performed a BLAST search against the BAC-based assembled genomic sequences obtained from all of the above contigs (unpublished data). A total of 17 sequences (a-q shown in Fig. 5) were detected within the different MTP BAC clones from 17 contigs that were physically located within the above three deletion bins. In particular, eight of these contigs were found to carry at least three Ty3/gypsy-type sequences ( $C R W$ or CCS1-R11H-2), indicating a significant accumulation of Ty3/gypsy retrotransposons in these genomic regions, which is consistent with results reported for other wheat chromosomes [51, 53]. The results obtained from the mapping and sequencing of these contigs clearly indicate that they contain a portion of the centromeric region of wheat chromosome 6B (Fig. 5). Contig CTG125 (0.5 Mb), located proximally on the short arm of chromosome $6 \mathrm{~B}$, contains eight centromere-specific sequences, whereas six and four centromere-specific sequences were found within the two contigs CTG3378 (1.0 Mb) and CTG3387 $(0.8 \mathrm{Mb})$, which mapped to the proximal region of the long arm (Fig. 5; Additional file 5). Many centromeric sequences were detected within other contigs (CTG14, CTG2884 and CTG9 on 6BS [bin C-6BS1, $40 \mathrm{cR}$ from the boundary between $6 \mathrm{BS}$ and 6BL], and CTG287 on 6BL [bin 6BL12, $48.6 \mathrm{cR}$ from the boundary]) (Fig. 5; Additional file 5). These results suggest that the genomic region from contig CTG14 to contig CTG287, with an estimated physical size of $25.4 \mathrm{Mb}$ (10 contigs with $13.0 \mathrm{Mb}$ on $6 \mathrm{BS}$ and 14 contigs with $12.4 \mathrm{Mb}$ on $6 \mathrm{BL}$ ), might correspond to the centromere of wheat chromosome $6 \mathrm{~B}$. However, we did not find any contigs with dominant satellite tandem repeats [51] in our physical map, although we detected sequences for the centromeric satellite-like repeat in CTG2884 on 6BS. A possible explanation for this observation is that the postulated 'centromere core region' with dominant satellite tandem repeats was not cloned or assembled because of its highly repetitive sequence composition.

A shift in the position of the centromere since the divergence of wheat and rice has been suggested based on comparisons between wheat chromosome $3 \mathrm{~B}$ and rice chromosome 1 [51]. To dtermine whether the centromeric shift occurred between chromosome $6 \mathrm{~B}$ and rice chromosome 2 , we investigated the relationships of orthologous genes in the centromeric region between wheat $6 \mathrm{~B}$ and rice 2 . A total of 54 genic markers were mapped on the contigs at the centromeric deletion bins (C-6BS1, C-6BL12 and 6BL12), of which 47 markers were associated with pericentromeric rice genes on chromosome 2 (Fig. 5). This result supports a conservation of the centromeric region, as shown in a previous study 
[54]. The centromeric region, from contig CTG307 (C-6BS1) to contig CTG482 (C-6BL12), was interposed between the genic markers TNAC8059 (CTG307 on C-6BS1) and GZ6H037 (CTG2358 on C-6BL12) (Fig. 5; Additional file 5). These two markers aligned to rice genes Os02g0241600 and Os02g0244700, respectively, on the short arm and at a position $5.6 \mathrm{Mb}$ distal to the centromere of rice chromosome 2 (Fig. 5). This suggests that the shift in the position of the centromere occurred between wheat $6 \mathrm{~B}$ and rice 2 , as observed between wheat $3 \mathrm{~B}$ and rice 1 .

\section{Distribution of genes along wheat chromosome 6B}

Genes are not evenly distributed along wheat chromosomes [18, 19, 55, 56]. After the removal of redundant markers based on sequence similarity, we discovered 875 markers derived from independent genes (378 on 6BS and 497 on $6 \mathrm{BL}$ ), which were able to anchor 322 contigs (137 on $6 \mathrm{BS}$ and 185 on $6 \mathrm{BL}$ ). To further extend the substantial gene content on chromosome 6B, we applied the gene models from the survey sequence data [15]. The sequence contigs assembled from the $6 \mathrm{~B}$ survey sequencing were mapped to the corresponding BAC contigs using their sequence similarities with the WGP tag sequences by BLAST. As a result, 50.9 and $61.1 \%$ of 6BS tags and 6BL tags were mapped on 21,339 IWGSC 6BS sequence contigs $(80.5 \mathrm{Mb})$ and 29,957 IWGSC 6BL sequence contigs (70.1 Mb). Finally, we mapped 1696 annotated genes on the survey sequence contigs to the physical map. After removal of redundancy, 1140 genes were integrated into the analysis based on the survey sequence data. In total, 2015 independent genes (859 on $6 \mathrm{BS}$ and 1156 on $6 \mathrm{BL}$ ) were assigned to 395 contigs (170 on $6 \mathrm{BS}$ and 225 on $6 \mathrm{BL}$ ). Based on the chromosomal locations of these genes on the contigs aligned along the RH map (Additional file 5), we obtained insight into the gene distribution pattern along chromosome 6B (Fig. 6a). The calculation of the numbers of genes and physical sizes of the gene containing contigs revealed an average gene density with similar values of 3.54 and 3.57 gene/ $\mathrm{Mb}$ for the two chromosome arms of $6 \mathrm{BS}$ and 6BL, respectively. However, the average gene densities for each contig were different; they ranged from 0.25 to 26.72 genes/Mb that were observed on the pericentromeric contig CTG42 on 6BS and the telomeric contig CTG3358 on $6 \mathrm{BL}$, respectively. The gene densities tended to increase from the centromeric to telomeric regions along chromosome 6B (Fig. 6a). This result was similar to the gene distribution on chromosomes $1 \mathrm{~B}$ and $3 \mathrm{~B}$ based on information obtained for deletion bins or the currently completed genomic sequences $[18,19,55,56]$.

On chromosomes $1 \mathrm{~B}$ and $3 \mathrm{~B}$, the distribution of syntenic genes had no impact on the overall gene-density gradient $[18,19,55]$. We found that 1538 of 2015 genes assigned on chromosome $6 \mathrm{~B}$ had orthologs on rice chromosome 2, B. distachyon chromosome 3 or sorghum

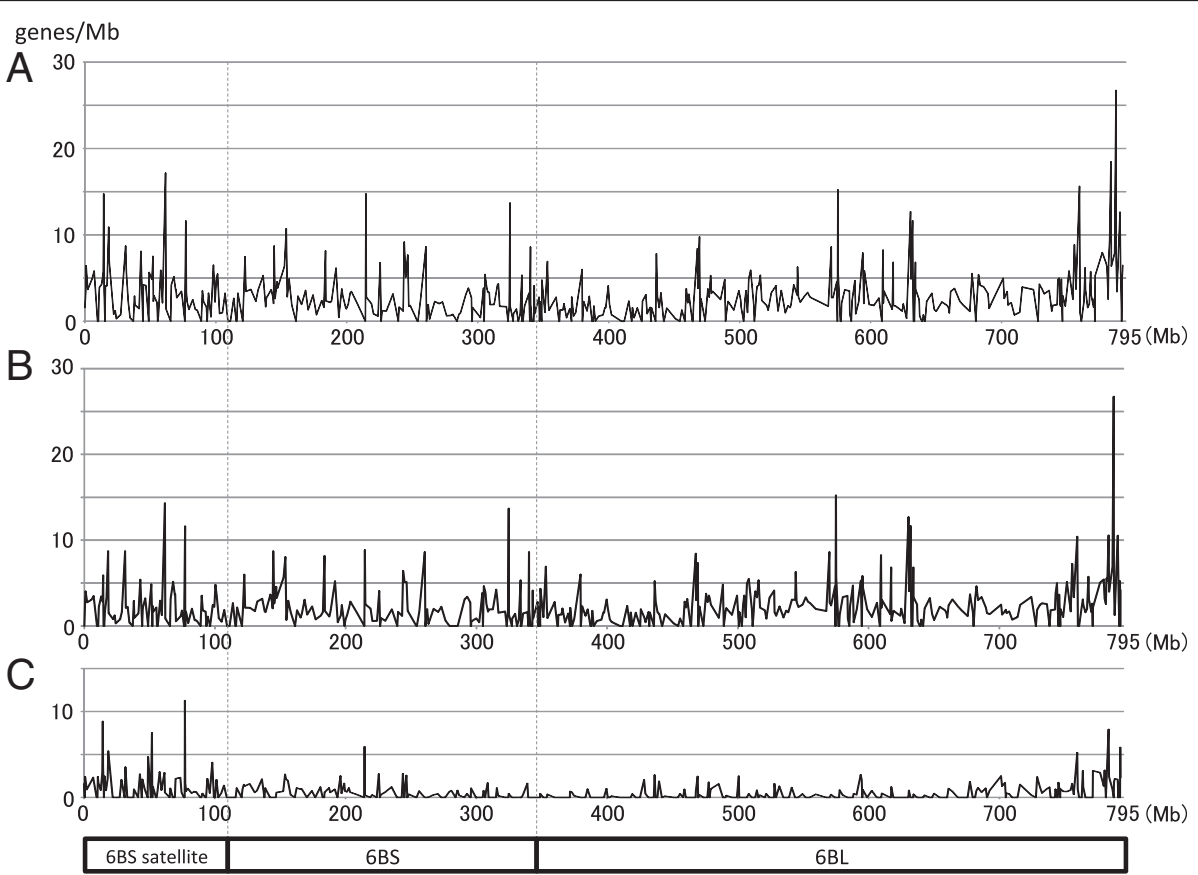

Fig. 6 Gene density of each contig on chromosome 6B. The distribution patterns of gene density on each of the 480 contigs on chromosome 6B are indicated in genes/Mb. a The pattern of all 2015 genes assigned on the physical map. $\mathbf{b}$ The pattern of 1538 syntenic genes, compared to those of rice chromosome 2, B. distachyon chromosome 3 or sorghum chromosome 4 . c The pattern of 477 nonsyntenic genes 
chromosome 4 and the remaining 477 genes were nonsyntenic. The distribution patterns of the nonsyntenic group on 6BS showed a clear gradient of gene density, becoming increasingly dense from centromere to telomere, whereas there was no discernable gradient pattern for the syntenic group (Fig. $6 \mathrm{~b}$ and $\mathrm{c}$ ). This shows that the gradient of gene density along 6BS is due to the presence of nonsyntenic genes, which was consistent with the general pattern of gene density on wheat chromosomes $1 \mathrm{~B}$ and $3 \mathrm{~B}[18,19$, 55]. In contrast, on 6BL, both the nonsyntenic and syntenic genes increased in density from centromere to telomere (Fig. $6 \mathrm{~b}$ and $\mathrm{c}$ ). This result suggests that the general pattern discussed above may not apply to 6BL. However, our result did not completely cover the positions of the genes on the 6B physical map; we used 2015 genes, representing only half of the 3746 high-confidence genes annotated on chromosome $6 \mathrm{~B}$ based on the IWGSC survey sequencing data [15]. Completion of the genomic sequencing and gene annotation will reveal the gene organization on chromosome $6 \mathrm{~B}$ more definitively.

\section{Local rearrangements on wheat chromosome 6B}

Previous comparative studies have demonstrated a syntenic and evolutionary relationship between the wheat homoeologous group 6 chromosomes and rice chromosome 2 (Os02), B. distachyon chromosome 3 (Bradi3) or sorghum chromosome 4 (Sb04) [42, 57-59]. Of the 2015 genes used in the present study for chromosome $6 \mathrm{~B}$, 1150,1415 and 1172 genes were syntenic with those of Os02, Bradi3 and Sb04, respectively, revealing strong genome colinearity between wheat and other grass species (Figs. $7 \mathrm{a}-\mathrm{c}$, respectively). However, the present study identified several specific genome rearrangements caused by events, such as inversions or translocations, as well as a lack of colinearity resulting from insertions and deletions.

Two inversions (188.3-226.9 and 230.2-267.1 Mb on the physical map, as shown in Additional file 5) were detected between wheat 6BS and the three syntenic chromosomes, Os02, Bradi3 and Sb04 (Table 6; Additional file 7), providing strong evidence for evolutionary events specific to the wheat lineage. However, between chromosome $6 \mathrm{~B}$ and rice Os2, two additional inversions were observed on the long arm (516.9-555.0 and 632.1-650.0 Mb), one of which (1205.7-1286.0 cR) was also identified between wheat and B. distachyon (Table 6; Additional file 7A and $\mathrm{B})$. This finding suggests that the inversion involving chromosomal region 516.9-555.0 Mb occurred recently, after the divergence of Brachypodieae and Triticeae. Interestingly, the other inversion on the long arm (632.1$650.0 \mathrm{Mb}$ ) shared by wheat chromosome $6 \mathrm{~B}$ and Bradi3 but not observed on Os02 was detected between chromosome 6D of Ae. tauschii and Os02 (Luo et al. [60]), suggesting that this event occurred after the divergence of
Ehrhartoideae (Oryzae) and Pooideae (Brachypodieae and Triticeae) and before the divergence of Pooideae. A comparison of wheat chromosome $6 \mathrm{~B}$ with Bradi3 indicated several complex rearrangements that appeared to have occurred within the genomic region of its long arm ranging from the deletion bin 6BL9 to the proximal two-fifth part of 6BL14 (Table 6; Additional file 5). This region included several translocations and inversions (602.5-713.8 Mb on the physical map), as well as a reciprocal translocation between the two regions on 602.5-713.8 and 715.8786.4 Mb. These translocations might be Brachypodieaespecific (Table 6; Additional file 7B) because the synteny between $6 \mathrm{~B}$ and $\mathrm{Os} 02$ in this region was well conserved (Additional file 7A).

A comparison of wheat chromosome 6B with Sb04 led to the discovery of one large inversion (559.5-630.8 Mb on the physical map) of the long arm 6BL (Table 6; Additional file $7 \mathrm{C}$ ). Because wheat $6 \mathrm{~B}$ and $\mathrm{Os02}$ or Bradi3 share the same inversion relative to $\mathrm{Sb04}$, this chromosomal structural change might be unique to the sorghum lineage. We discovered two reciprocal translocations between wheat 6B and Sb04 (Table 6; Additional file $7 \mathrm{C}$ ), which corresponded exactly to the two Pooideae-specific inversions described above (Table 6; Additional file 7A).

These data allow us to propose the following scenarios for rearrangements on the long arm during the evolution of grass species (Fig. 8): 1) the chromosomal region corresponding to $516.9-650.0 \mathrm{Mb}$ on the physical map was inverted after the divergence of Panicoideae (Sorghum) and Ehrhartoideae/Pooideae; 2) the distal part of the first inversion, 632.1-650.0 Mb, was inverted again after the divergence of Ehrhartoideae and Pooideae; and 3) the proximal region of the first inversion, 516.9-555.0 Mb, was recently inverted after the divergence of Brachypodieae and Triticeae. These results also indicate that chromosomal rearrangements occurred frequently in the distal regions of the homologous chromosomes, including wheat 6B.

\section{Disruption of synteny in the proximal region of wheat chromosome 6BL}

A large rearrangement in the proximal region (deletion bins, C-6BL12, 6BL12 and 6BL13; Fig. 7d; 340.2-421.8 Mb in Additional file 7; Additional file 5) was observed between $6 \mathrm{~B}$ and the Sb04, Os02, and Bradi3 chromosomes; that is, fewer syntenic genes were mapped to this genomic region. Such an interruption of synteny in the proximal region of wheat homoeologous group 6 chromosomes relative to rice chromosome Os02 has been previously described by the mapping of orthologous genes [42]. It has also been documented between chromosome 6D of Ae. tauschii and Os02 based on high-resolution physical mapping [60]. Using the genomic sequences of the MTP BAC clones in this region (unpublished results), we estimated the number of genes 


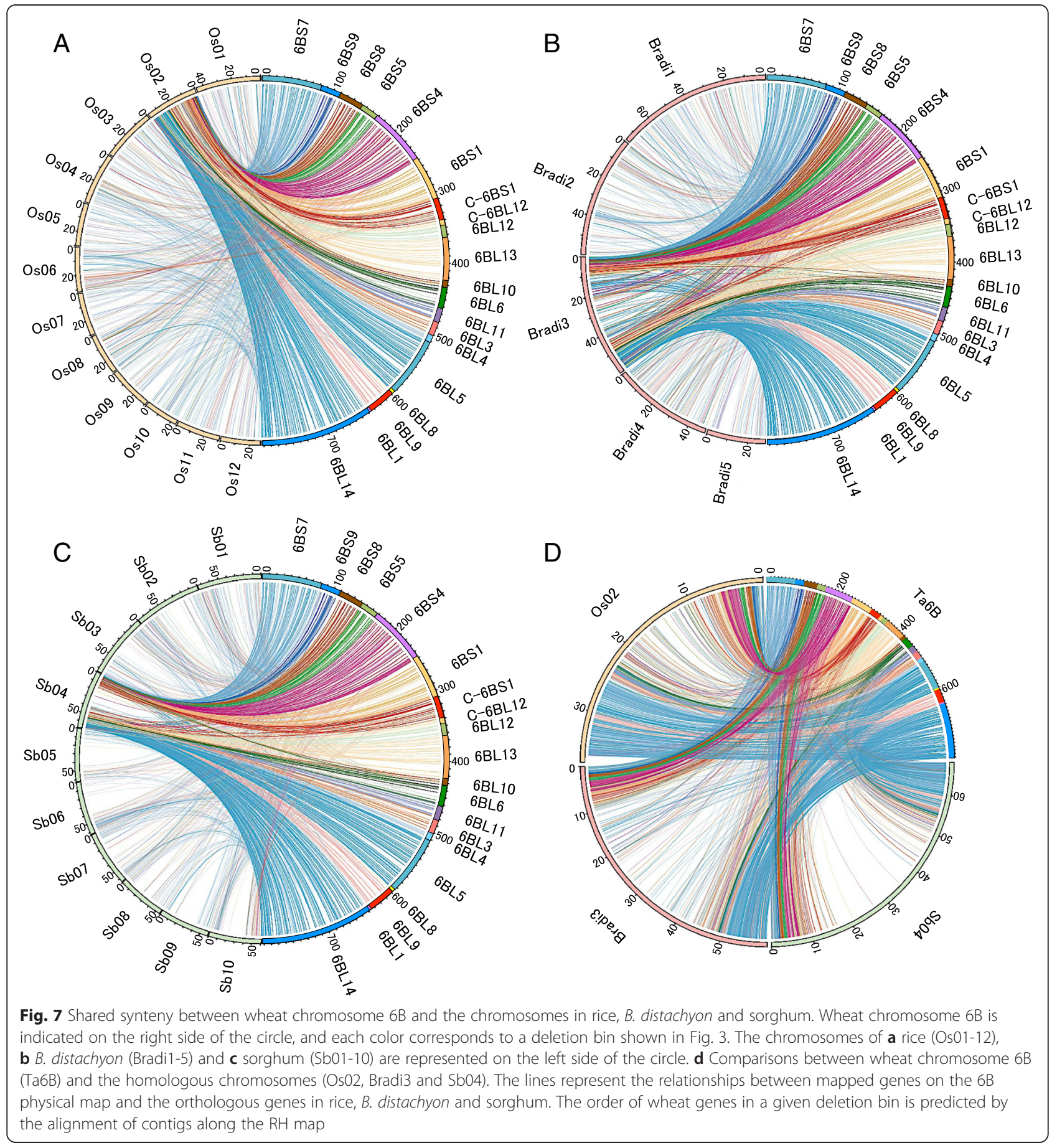

in these bins that were homologous to the rice genes by a BLAST analysis. From 864 MTP clones $(85 \mathrm{Mb})$ in the three bins, we identified 517 gene sequences homologous to the rice genes. We found 70 and 69 gene sequences that were homologous to Os01 and Os02, respectively, and 25-56 gene sequences were derived from other chromosomes in rice. We investigated the colinearity of the 69 sequences that were homologous to Os02 based on their positions on Os02, but we failed to detect a certain level of colinearity. The estimated proportion of nonsyntenic genes in this region was very high (87\%; 449 in 517 sequences) compared with the proportion (57\%) in the entire chromosome $6 \mathrm{~B}$ based on our survey sequence data, as noted previously [24]. Furthermore, a recent genome sequencing study reported that the proportion of nonsyntenic genes in the proximal region of chromosome $3 \mathrm{~B}$ was $28 \%$, and even in distal regions, the proportions were 44 and $53 \%$ [56]. These results suggest that the 
Table 6 Genomic regions containing chromosome rearrangements between 6B and its homologous chromosomes in other species

\begin{tabular}{|c|c|c|c|c|c|}
\hline (position on RH map) & (estimated size $\left.{ }^{\mathrm{a}}\right)$ & Rearrangement & (size) & $\begin{array}{l}\text { B. distachyon } \\
\text { chromosome } 3 \\
\text { (size) }\end{array}$ & $\begin{array}{l}\text { Sorghum } \\
\text { chromosome } 4 \\
\text { (size) } \\
\end{array}$ \\
\hline 6BS: 188.3-226.9 Mb & 6BS: $355.7-422.8 c R$ & Inversion & Os02g0169900-Os02g0184500 & $\begin{array}{l}\text { Bradi3g05260.1- } \\
\text { Bradi3g06250.1 }\end{array}$ & $\begin{array}{l}\text { Sb04g004700.1- } \\
\text { Sb04g005730.1 }\end{array}$ \\
\hline$(355.7-422.8 c R)$ & (41.9 Mb) & & (0.93 Mb) & (0.85 Mb) & (1.1 Mb) \\
\hline 6BS: 230.2-267.1 Mb & 6BS: 424.0-511.9cR & Inversion & Os02g0185500-Os02g0200000 & $\begin{array}{l}\text { Bradi3g06260.1- } \\
\text { Bradi3g07370.1 }\end{array}$ & $\begin{array}{l}\text { Sb04g005790.1- } \\
\text { Sb04g006830.1 }\end{array}$ \\
\hline$(424.0-511.9 c R)$ & (40.4 Mb) & & (0.84 Mb) & (0.94 Mb) & $(1.2 \mathrm{M})$ \\
\hline 6BL: 516.9-555.0 Mb & 6BL: 1205.7-1286.0cR & Inversion & Os02g0644100-Os02g0680400 & $\begin{array}{l}\text { Bradi3g50100.1- } \\
\text { Bradi3g51730.1 }\end{array}$ & \\
\hline$(1205.7-1286.0 c R)$ & (43.8 Mb) & & (1.9 Mb) & (1.4 Mb) & \\
\hline 6BL: 632.1-650.0 Mb & 6BL: $1400.4-1432.3 c R$ & Inversion & Os02g0739000-Os02g0751800 & & \\
\hline$(1400.4-1432.3 c R)$ & (22.3 Mb) & & (0.73 Mb) & & \\
\hline 6BL: 602.5-713.8 Mb & 6BL: 1373.4-1479.1cR & Translocation (inversion) & & $\begin{array}{l}\text { Bradi3g56180.1- } \\
\text { Bradi3g60210.1 }\end{array}$ & \\
\hline$(1373.4-1479.1 \mathrm{cR})$ & (98.8 Mb) & & & (3.0 Mb) & \\
\hline 6BL: 715.8-786.4 Mb & 6BL: 1494.5-1545.2cR & Translocation & & $\begin{array}{l}\text { Bradi3g52910.1- } \\
\text { Bradi3g56110.1 }\end{array}$ & \\
\hline$(1494.5-1545.2 \mathrm{cR})$ & (70.6 Mb) & & & (2.4 Mb) & \\
\hline 6BL: 516.9-555.0 Mb & 6BL: 1205.7-1286.0cR & Translocation & & & $\begin{array}{l}\text { Sb04g031850.1- } \\
\text { Sb04g033430.1 }\end{array}$ \\
\hline$(1205.7-1286.0 c R)$ & (43.8 Mb) & & & & (1.6 Mb) \\
\hline 6BL: 559.5-630.8 Mb & 6BL: 1293.0-1398.2cR & Inversion & & & $\begin{array}{l}\text { Sb04g028600.1- } \\
\text { Sb04g031680.1 }\end{array}$ \\
\hline$(1293.0-1398.2 c R)$ & (72.4 Mb) & & & & (2.9 Mb) \\
\hline 6BL: 632.1-650.0 Mb & 6BL: $1400.4-1432.3 c R$ & Translocation & & & $\begin{array}{l}\text { Sb04g027710.1- } \\
\text { Sb04g028500.1 }\end{array}$ \\
\hline$(1400.4-1432.3 c R)$ & (22.3 Mb) & & & & (0.96 Mb) \\
\hline
\end{tabular}

${ }^{\mathrm{a}}$ The size of the genomic region on chromosome $6 \mathrm{~B}$ is estimated based on the cumulative size of the BAC contigs positioned in the corresponding $\mathrm{RH}$ map region

interruption was unusual; it was not due to the simple integration of chromosomal segments from a rice chromosome other than Os02 into this region but rather to the abundant and random accumulation of nonsyntenic genes that might disrupt the synteny between wheat chromosome $6 \mathrm{~B}$ and rice chromosome 2. Although we do not currently have any data to explain the mechanisms underlying the disruption in colinearity in this region, it might be due to structural differences in the centromeric region, including the previously described centromere shifting.

Large interruptions of approximately $30-40 \mathrm{Mb}$ of the genomic region of Bradi3 and Sb04 were also found (Fig. 7d; Additional files 7B and C). The interruption between $6 \mathrm{~B}$ and Bradi3 revealed characteristics of the chromosomal structures of Bradi3, which was postulated to correspond to three rice chromosomes (Os02, Os08 and Os10), and its pericentromeric $10-45 \mathrm{Mb}$ region was syntenic with Os08 and Os10 [6]. Therefore, the interruption between $6 \mathrm{~B}$ and Bradi3 resulted from the insertion of other chromosomal segments in Bradi3. The cause of the interruption between $6 \mathrm{~B}$ and $\mathrm{Sb} 4$ remains unclear, although the small number of annotated gene in this $30-\mathrm{Mb}$ genomic region corresponding to the proximal region of Sb04 [7] is estimated to be one of the causes.

In summary, we could infer the structural features of chromosome $6 \mathrm{~B}$ with high resolution based on the the physical localizations of genic markers obtained by $\mathrm{RH}$ mapping (Fig. 3e). The results provide information to understand the molecular and biological mechanisms underlying the genomic divergence and chromosomal evolution of wheat.

\section{Conclusions}

Here, we provide a BAC-based physical map of wheat chromosome 6B with an estimated size of $833.8 \mathrm{Mb}$ that covers $91 \%$ of the chromosome, which allowed us to select MTP BAC clones for the map-based genomic sequencing of chromosome 6B. The development of 2860 anchor markers and the construction of a highresolution RH map permitted the successful localization 


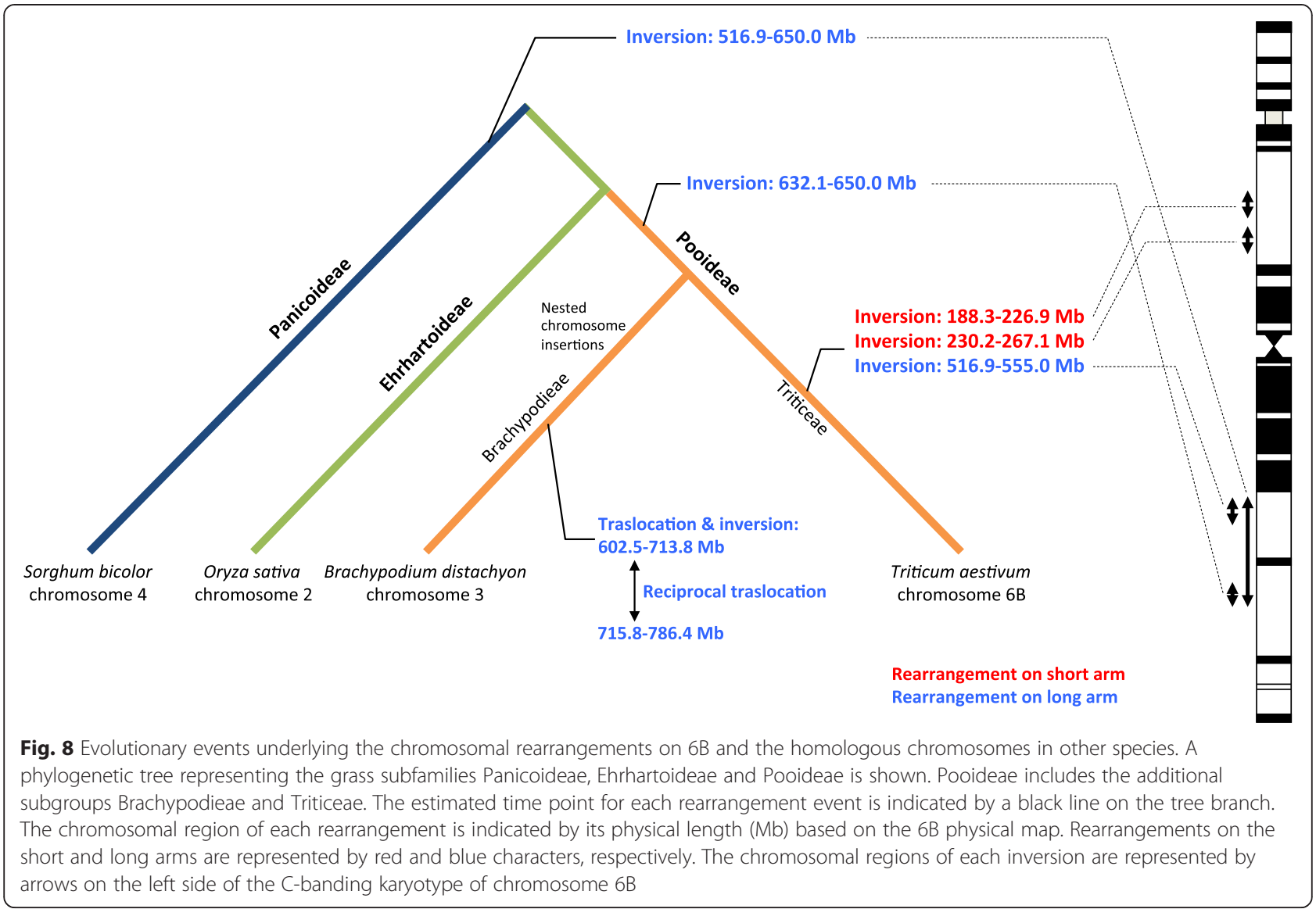

of 480 of the assembled contigs to their chromosomal regions, representing $87 \%(794.8 \mathrm{Mb})$ of the entire chromosome. The establishment and analysis of the integrated physical map also led to the discovery of several important features of chromosome $6 \mathrm{~B}$, including the fine chromosomal localization and organization of Nor-B2 and the centromere, the gene distribution patterns and the evolutionary history of chromosomal rearrangements among grass species. Furthermore, the use of marker information from syntenic genes led to the identification of chromosomal regions that were highly conserved or frequently rearranged, which is useful for our understanding of the complexity of genome evolution in wheat.

\section{Methods}

\section{Construction of BAC libraries}

The short and long arms of wheat chromosome $6 \mathrm{~B}$ were isolated from a double ditelosomic line $(2 n=40+2 \mathrm{t} 6 \mathrm{BS}+$ $2 \mathrm{t} 6 \mathrm{BL}$ ) of CS [30] that was obtained from NBRP-Wheat Japan (accession number LPGKU0038). Aqueous suspensions of intact mitotic chromosomes were prepared from synchronized root tips of young seedlings as described by Vrána et al. [61], and both arms of 6B were purified by flow cytometry [24]. The purity of the flow-sorted fractions was determined by FISH using probes for GAA microsatellites and telomeric repeats as described by Janda et al. [62]. A total of 5,200,000 6BS arms and 5,150,000 6BL arms were sorted by flow cytometry, embedded in agarose miniplugs (each containing approximately 200,000 arms) and used to construct BAC libraries according to Šimková et al. [63]. Briefly, high-molecular-weight DNA was partially digested with HindIII and subjected to two rounds of size selection using pulsed-field gel electrophoresis. The DNA was electroeluted from the gel and ligated into the pIndigoBAC-5 vector (Epicentre, Madison, WI, USA). The recombinant vector was used to transform Escherichia coli DH10B competent cells (Invitrogen, Carlsbad, CA, USA). The libraries were ordered into 384-well plates filled with freezing medium consisting of 2YT, $6.6 \%$ glycerol and $12.5 \mu \mathrm{g} / \mathrm{ml}$ chloramphenicol and stored at $-80{ }^{\circ} \mathrm{C}$. The average insert size was estimated based on an analysis of 240 randomly selected BAC clones in each library.

\section{Fingerprinting by whole-genome profiling}

Fingerprinting of chromosome arm 6BS- and 6BLspecific BAC libraries was performed by using a WGP ${ }^{\mathrm{rm}}$ method that was developed based on NGS technology to establish the chromosome physical maps [31]. Using 41,472 clones of 6 BS (plate no. 41-148) and 49,920 
clones of 6BL (plate no. 69-198), a multi-dimensional pool of BAC DNAs with a high concentration and low E. coli level was developed by Amplicon Express, Inc. (Pullman, WA, USA). The pooled BAC DNAs were subjected to sample preparation and sequencing by KeyGene N.V. (Wageningen, The Netherlands): a) digestion with the restriction enzymes EcoRI and Msel, ligation of sequencing adaptors containing sample identification tags and PCR amplification; b) pooling of the PCR products; c) cluster amplification; and d) sequencing from the EcoRI side using the HiSeq2000 sequencer (Illumina, San Diego, CA, USA) with a read length of 81 nucleotides. The high-quality reads were used for WGP data processing, which included the following steps: a) deconvolution, i.e., assignment of sequencing reads to individual BACs in the pools; b) assignment of WGP tags to BACs based on the deconvoluted reads; and c) filtering of WGP tags and BACs using various quality control measures to minimize noise.

\section{BAC contig assembly}

The assembly of BAC clones was performed using FPC software [33], which was improved by KeyGene N.V. to be capable of processing the WGP data. The assembly procedure was based on the previously described guidelines for physical map assembly of IWGSC and chromosome $3 \mathrm{~B}$ physical mapping $[20,32]$ as shown below: a) the initial assembly was performed using incremental contig-building with a cut-off of $1 \mathrm{e}^{-75}$; b) a single-to-end and end-to-end merge was conducted using the Match 1 parameter by increasing the cut-off $\left(1 \mathrm{e}^{-5}\right.$ at each step) to a final cut-off of $1 \mathrm{e}^{-05}$; c) the DQer function was used at each cut-off step to separate all of the contigs containing more than $10 \%$ of Questionable $(\mathrm{Q})$ clones with the Step 3 parameter; d) finally, a single optimal cut-off value was determined. The following parameters were used for the WGP analysis: a band size (CB unit, average distance between tags) of 5220 and 5075 for 6BS and $6 \mathrm{BL}$, respectively; a gel length (corresponding to the number of unique tags) of 120,913 and 112,559 for 6BS and 6BL, respectively; a FromEnd value (corresponding to half of the average number of tags per BAC) of 12; and a tolerance of 0 . The MTP was selected using the FPC MTP module [64].

\section{Elimination of low-confidence BAC contigs}

To detect BAC clones derived from other chromosomal DNAs that contaminated our preparation, a BLASTN [65] search was performed against the survey sequence of wheat [15] using the WGP tag sequences on the BAC clones as queries with thresholds of $95 \%$ coverage and $95 \%$ identity. First, we identified WGP tags with high similarity to each chromosome group. Next, we compared the number obtained for the group 6 chromosomes and the largest number among the other chromosome groups.
If the number obtained for one of the other groups was larger (more than four) than the number obtained for group 6 , we judged that the BAC clone was derived from the other chromosomes. Contigs that included BAC clones occupying more than $66 \%$ of the total number of BAC clones within a contig were eliminated from further experiments and were labeled as low-confidence contigs.

\section{Marker selection and development}

We used several sources to develop markers used in this study, which were mainly divided into three groups. The first group was the markers that genetically mapped to chromosome $6 \mathrm{~B}$ and deposited in the public databases: GrainGenes CMap and NBRP-Wheat, Japan. From these databases, we selected 208 PCR-based markers such as STS-RFLP and SSR markers. Additionally, 751 PLUG markers [34, 35] (http://plug.dna.affrc.go.jp/) were selected based on synteny of wheat group- 6 chromosomes to rice chromosome 2 .

The second group consisted of markers derived from wheat and barley expressed sequences, i.e., full-length cDNAs [66, 67] and ESTs (Ta\#56: wheat ESTs deposited in build \#56 of the NCBI UniGene dataset; http:// www.ncbi.nlm.nih.gov/unigene). In the Genome Zipper [36], we found 2304 non-redundant reads by NGS mapped on chromosome $6 \mathrm{H}$ of barley. We identified wheat ESTs homologous to the above barley $6 \mathrm{H}$ reads by BLAST (390 markers). Additionally, we selected following wheat sequences as marker sources: a) full-length cDNAs with $\geq 70 \%$ coverage and $\geq 50 \%$ identity with the syntenic genes common to Os02, Bradi3 and Sb04 (orthologous set, 905 markers); b) full-length cDNAs and ESTs with more than 80 and $85 \%$ identities to genes of Os02 and Bradi3, respectively (predicted orthologous set, 482 FLcDNA and 309 EST markers); and c) full-length cDNAs with more than $85 \%$ identity with Bradi3 genes listed in the barley $6 \mathrm{H}$ Genome Zipper (predicted orthologous set from B. distachyon, 133 markers).

The third group contained ISBP markers, which were developed from the survey sequence data for $6 \mathrm{BS}$ and 6BL [15] according to Paux et al. [28]. Among the highconfidence primer set identified (Kaneko et al. in preparation), 2000 sets were selected based on the frequency of the number of junctions between the transposable element subfamilies.

To determine the chromosomal locations of contigs that lacked the above anchors, additional PCR markers such as ISBP and SSR were developed using the genomic sequences of the MTP BAC clones and were used for RH mapping. High-confidence ISBP markers were developed as described by Paux et al. [28], and Primer3 was used to design SSR primers at positions flanking the microsatellite sequences $(>4$ units of di-, tri- and tetra- 
nucleotides) predicted by Perl scripts. To develop 6Bspecific markers, a BLASTN search using the primer sequences as queries was performed against the wheat survey sequence data [15]. Primer pairs with complete identity to the only chromosome $6 \mathrm{~B}$ sequences were 1076 ISBP (426 for 6BS and 650 for 6BL contigs) and 1163 SSR (447 for 6BS and 716 for 6BL contigs) markers, from which 79 ISBP (37 for 6BS and 42 for $6 \mathrm{BL}$ contigs) and 74 SSR (38 for $6 \mathrm{BS}$ and 36 for $6 \mathrm{BL}$ contigs) were used for anchoring.

\section{PCR screening of BAC libraries}

Prior to BAC library screening, the PCR-based molecular markers were tested to determine their quality and specificity to chromosome 6B. For the PCR amplification, total DNA was extracted from CS, the Nullisomic-6B Tetrasomic-6A (N6BT6A) line and M808 using standard procedures, and the DNAs of the sorted chromosome arms 6BS and 6BL were amplified by multiple displacement amplification using the illustra ${ }^{\text {Ta }}$ GenomiPhi V2 DNA Amplification Kit (GE Healthcare Bio-Science Corp., Piscataway, NJ, USA). PCR reactions were performed using Quick Taq Solution (Toyobo, Osaka, Japan) according to the manufacturer's recommendations. Touchdown PCR amplification was performed as follows: initiation at $94{ }^{\circ} \mathrm{C}$ for $2 \mathrm{~min}, 10$ cycles $\left(94{ }^{\circ} \mathrm{C}\right.$ for $20 \mathrm{~s}, 65{ }^{\circ} \mathrm{C}$ minus $0.5^{\circ} \mathrm{C}$ each cycle for $20 \mathrm{~s}, 72{ }^{\circ} \mathrm{C}$ for $\left.20 \mathrm{~s}\right), 20$ cycles $\left(94{ }^{\circ} \mathrm{C}\right.$ for $2 \mathrm{~min}, 60^{\circ} \mathrm{C}$ for $20 \mathrm{~s}, 72{ }^{\circ} \mathrm{C}$ for $20 \mathrm{~s}$ ) and termination at $72{ }^{\circ} \mathrm{C}$ for $1 \mathrm{~min}$. The amplified products were analyzed by using a MultiNA microchip electrophoresis system (Shimadzu, Kyoto Japan) according to the manufacturer's recommendations.

Screening was performed using the BAC DNA pools, which were created from the above BAC libraries for $6 \mathrm{BS}$ and $6 \mathrm{BL}$, comprising 57,600 and 76,032 BAC clones, respectively. The BAC DNAs were extracted from threedimensional (plate, row, column) pooled BACs and purified using the alkaline lysis method [68]. Each pool contained $100 \mu \mathrm{g}$ of BAC DNA. The PCR reaction was performed in $15 \mu \mathrm{L}$ containing $30 \mathrm{ng}$ of BAC pooled DNA, $0.2 \mu \mathrm{M}$ of each primer and $5 \mu \mathrm{L}$ of GoTaq Green Master Mix (Promega, Madison, WI, USA). Amplification was initiated at $94{ }^{\circ} \mathrm{C}$ for $1 \mathrm{~min}$, followed by $35 \mathrm{cy}$ cles of $94{ }^{\circ} \mathrm{C}$ for $30 \mathrm{~s}, 60{ }^{\circ} \mathrm{C}$ for $1 \mathrm{~min}, 72{ }^{\circ} \mathrm{C}$ for $1 \mathrm{~min}$, and termination at $72{ }^{\circ} \mathrm{C}$ for $1 \mathrm{~min}$. The resulting fragments were electrophoresed in a $2 \%$ agarose gel.

\section{RH mapping}

The $\mathrm{RH}$ panel of chromosome $6 \mathrm{~B}$ was produced by Watanabe et al. (in preparation). We selected 355 lines as the $\mathrm{RH}$ panel based on the genotypes determined using the $216 \mathrm{~B}$-specific SSR markers. In addition to the $\mathrm{RH}$ panel, 21 chromosome deletion lines (9 lines for 6BS and 12 lines for 6BL) [39] were used for the approximate mapping of markers to each chromosome bin. CS and the N6BT6A line were included as positive and negative controls, respectively, for the amplification of the PCR markers. The seeds of CS (LPGKU2269), the N6BT6A line (LPGKU0075) and the 21 chromosome deletion lines (LPGKU1247-1267) were obtained from NBRPWheat, Japan.

To genotype the RH panel and chromosome deletion lines, we used 653 markers that were anchored on 462 contigs and defined based on their specificity for chromosome 6B using the N6BT6A line. The presence/ absence of the markers was analyzed by agarose gel electrophoresis.

The data obtained for the 653 markers were used to group and order the markers in Carthagene version 1.3.beta [69]. The linkage group was determined by using the group command with a two-point LOD threshold of 4.0 and a maximum distance of $100 \mathrm{cR}$. The heap command was used to identify the map with the highest likelihood. The mapocb command was used to calculate the number of obligate breaks.

\section{Calculation of gene density along chromosome 6B}

We used two gene information sources to calculate the gene density along chromosome 6B. First, we extracted markers representing independent genes from the DNA markers used for the anchoring BAC clones. Redundancy in the marker set was removed based on the sequence similarity using CAP3 [70] with $98 \%$ identity. BLASTX search for the resulting non-redundant markers were conducted to the rice, Brachypodium and sorghum genes with $\mathrm{E}$ value $<10^{-5}$. Then, markers hit to the genes on rOs02, Bardi3 and Sb04 were used for the further analysis. Second, we used gene models annotated from the survey sequencing data by IWGSC [15]. We mapped 731,925 and 998,825 WGP tags on 6BS and 6BL to IWGSC sequence contigs assembled from the $6 \mathrm{~B}$ survey sequencing [15] by megablast with default setting. Gene information on $6 \mathrm{~B}$ survey sequence contigs were assigned to BAC contigs based on the information of the WGP tags uniquely mapped to $6 \mathrm{~B}$ survey sequence contigs with $100 \%$ identity and $100 \%$ coverage. If the tags located in distant contigs were mapped on a same survey sequence contig, we discarded the hit information.

\section{Comparison of the virtual gene order based on the physical map with the syntenic chromosomes of other cereals}

To compare the gene order between $6 \mathrm{~B}$ and its homologous chromosomes, Os02, Bradi3 and Sb04, a BLASTX search with an E-value $<10^{-5}$ [65] was conducted against the protein sequences of the three grasses using nucleotide sequences of the aforementioned gene set. The positions of the genes on the $6 \mathrm{~B}$ physical map were 
compared with the physical genomic positions of the orthologous sequences on the homologous chromosomes in the three cereals. The graphical displays of the relationships between orthologous genes were drawn using Circos-0.67-7 software [71].

\section{Sequencing of MTP BAC clones}

BAC clones were sequenced using Roche GS FLX (Basel, Switzerland). BAC DNAs were prepared in a 96deepwell plate, extracted using the alkaline lysis method and purified with the MultiScreen Filter plate (Millipore, Billerica, MA, USA). After fragmentation with the Covaris Acoustic Solubilizer LE220 (Covaris, Woburn, MA, USA), the sequencing libraries were constructed using the NEBNext DNA Library Prep Master Mix Set for 454 (New England Biolabs, Ipswich, MA, USA) and tagged with Multiplex Identifier (MID) Adaptors (Roche, Basel, Switzerland). The tagged DNAs were subjected to size selection using agarose gel electrophoresis to obtain DNA fragments of the appropriate size (600-1200 bp) and then quantified using the Agilent 2100 Bioanalyzer (Agilent, Santa Clara, CA, USA). The resulting libraries were sequenced according to the manufacturer's recommendations. The resulting SFF files were split into separate files of each BAC clone based on MID by using the sffile program in SFF Tools (Roche, Basel, Switzerland). The split SFF files were used for de novo assembly with the GS De Novo Assembler v2.6 (Roche, Basel, Switzerland) with default parameters, and the BAC vector and E. coli genome sequences were trimmed.

\section{Sequence analysis}

To detect ribosomal RNA genes, a BLASTN search was performed against BAC sequences with E-values $<10^{-10}$. For use as queries, four rRNA gene sequences from wheat, 5S (accession no. 3IZ9), 5.8S (3IZ9), 18S (3IZ7) and 25S (3IZ9) [72], were obtained from the Protein Data Bank (PDB; http://www.pdb.org/pdb/home/home.do), and the IGS sequence (X07841) [44] was obtained from DDBJ/EMBL/GenBank (http://www.ddbj.nig.ac.jp).

To identify BAC clones containing centromeric sequences, a BLASTN search was run against the BAC sequences with E-value $<10^{-4}$, and a low-complexity filter was used. Eighteen centromeric sequences were used as queries: 15 probe sequences (KC290868KC290916) [50], the CCS1-R11H-2 gene (AB048245) [52], the rye centromere-specific marker (KF719092) and contig 310431 [51] of the 5xCS genome sequence [11].

\section{Availability of supporting data}

A genome browser of the physical map of the wheat chromosome $6 \mathrm{~B}$ is available from the Unite de Recherche Génomique Info website (https://urgi.versailles.inra.fr/gb2/ gbrowse/wheat_phys_pub/) and the Komugi Genome
Sequence Program web site (http://komugigsp.dna.affrc.go.jp/index.html). The other supporting data are included as additional files.

\section{Additional files}

Additional file 1: BAC fingerprinting by whole-genome profiling.

(PDF $35 \mathrm{~kb}$ )

Additional file 2: Distribution of the number of WGP tags per BAC clone in the libraries of $6 \mathrm{BS}$ and $6 \mathrm{BL}$. To fingerprint the BAC libraries with WGP, the number of tags on each clone was determined by deconvolution by assigning the sequence reads to individual BACs. The gray bars represent the number of eliminated BACs that were judged to be low quality based on the number of tags (less than 7 tags or more than 51 tags). The BAC clones represented by white bars were used for subsequent contig assembly. (PDF $50 \mathrm{~kb}$ )

Additional file 3: Final fingerprinting data used for contig assembly after BAC filtering. (PDF $33 \mathrm{~kb}$ )

Additional file 4: Features of the physical maps of chromosome arms $6 \mathrm{BS}$ and 6BL. (PDF $38 \mathrm{~kb}$ )

Additional file 5: Integrated data for the $\mathrm{RH}$ map and BAC contigs of chromosome $6 \mathbf{B}$. The markers, distance between markers and cumulative distance are provided for the $6 \mathrm{~B} \mathrm{RH}$ map. The marker location on the deletion bin is also indicated. The ordered contig data mapped on the $\mathrm{RH}$ map with each of the markers provide the contig name, contig size, number of BAC clones, number of MTP clones and names of the markers anchored in the same contig. The physical position of each contig is denoted by the cumulative contig size starting from the contig '6BS_CTG219' located on the end of 6BS. (XLSX 189 kb)

Additional file 6: Schematic of the NOR structure by comparing wheat Nor-B2 on chromosome 6B and rice NOR on chromosome 9.

The structure of the rice NOR on chromosome 9 is depicted according to Fujisawa et al. [49] with modifications. Wheat Nor-B2 is illustrated based on Fig. 4, showing the five contigs located around the Nor-B2 locus. Each rDNA unit is represented by a horizontal arrow in the illustration of rice and wheat. In the illustration of Nor-B2, ISBP and genic markers that mapped to the five contigs are indicated by white and black boxes, respectively. The junction sequences of the ISBP markers are indicated in parentheses below the marker name. The uncharacterized genomic region within the contigs is represented by a dashed horizontal line. (PDF 68 kb)

Additional file 7: Relationship of the gene positions between wheat chromosome 6B and the syntenic chromosomes of grass species. Dot plot of the best matches between genes on wheat chromosome $6 \mathrm{~B}$ and the syntenic chromosomes: (A) 6B vs. rice chromosome $2(\mathrm{Os} 02$ ); (B) $6 \mathrm{~B}$ vs. B. distachyon chromosome 3 (Bradi3); and (C) 6B vs. sorghum chromosome 4 (Sb04). The horizontal axis in the dot plots indicates the position of assigned genes on the $6 \mathrm{~B}$ physical map, and the vertical axis indicates the physical positions of genes on each syntenic chromosome. (PDF $259 \mathrm{~kb}$ )

\section{Abbreviations}

BAC: Bacterial artificial chromosome; BLAST: Basic Local Alignment Search Tool; cR: Centiray; EST: Expression sequence tag; FISH: Fluorescence in situ Hybridization; FPC: FingerPrinted Contigs; Gb: Gigabase; IGS: Intergenic spacer sequence; ISBP: Insertion site-based polymorphism; IWGSC: International Genome Sequencing Consortium; kb: Kilobase; Mb: Megabase; MTP: Minimal tiling path; NGS: Next-generation sequencing; NOR: Nucleolus organizer region; PCR: Polymerase chain reaction; PLUG: PCR-based Landmark Unique Gene; RFLP: Restriction fragment length polymorphism; RH: Radiation hybrid; RIL: Recombinant inbred line; rDNA: Ribosomal DNA; rRNA: Ribosomal RNA; STS: Sequence-tagged site; SSR: Simple sequence repeat; WGP: Whole Genome Profiling.

\section{Competing interests}

The authors declare that they have no competing interests. 


\section{Authors' contributions}

FK conducted the physical mapping, anchoring of the physical map in the $\mathrm{RH}$ and genetic maps, comparative genomic analysis, chromosome structural analysis and drafting of the manuscript. JW supervised the physical mapping and anchoring. HK and KK produced the MTP sequencing data as well as the BAC-end sequencing data. TT developed the primer pairs for gene-based and ISBP markers using 6B survey sequencing data. SK, WK, YH and HF handled the $6 \mathrm{~B}$ BAC libraries and produced the three-dimensional MTP pools. SK, SW and TS participated in the development of the PCR markers, $\mathrm{RH}$ panel and $\mathrm{Gc}$ panel, respectively. $\mathrm{CA}$ and $\mathrm{KH}$ analyzed the specificity of the PCR markers. JCMI, RO and ST conducted the genotyping and construction of the genetic map. JŠ, HŠ and JD prepared the purified fraction of the chromosome arms of $6 \mathrm{~B}$ and produced the $6 \mathrm{BS}$ and $6 \mathrm{BL}$ BAC libraries. YM, MH and YK contributed to the data management and analysis tools. MS, GI and TN produced the PLUG marker information. TRE participated in the plant line generation and seed multiplication. KS and YO generated the data for the full-length CDNAs and ESTs of barley and wheat, respectively. SN supervised the marker development and construction of the RH panels. TM acquired the funding and supervised the physical map construction. $\mathrm{HH}$ acquired the funding, supervised all of the analyses and drafted the manuscript. All authors have read and approved the final manuscript.

\section{Acknowledgments}

The authors wish to thank Etienne Paux for critical editing of the manuscript. This work was supported by the Ministry of Agriculture, Forestry, and Fisheries of Japan (Genomics for Agricultural Innovation, grant number KGS-1003 and -1004; Genomics-based Technology for Agricultural Improvement, NGB-1003), the Czech Science Foundation (award P501/12/G090), and the National Program of Sustainability of the Czech Republic (LO1204).

\section{Author details}

${ }^{1}$ Plant Genome Research Unit, National Institute of Agrobiological Sciences, Tsukuba 305-8602, Japan. ${ }^{2}$ Advanced Genomics Laboratory, National Institute of Agrobiological Sciences, Tsukuba 305-8602, Japan. ${ }^{3}$ Bioinformatics Research Unit, National Institute of Agrobiological Sciences, Tsukuba 305-8602, Japan. ${ }^{4}$ Laboratory of Plant Genetics, Graduate School of Agriculture, Kyoto University, Kyoto 606-8502, Japan. ${ }^{5}$ Cereal Science Research Center of Tsukuba, Nisshin Flour Milling Inc., Tsukuba 300-2611, Japan. 'aboratory of Plant Genetics, Graduate School of Agricultural Science, Kobe University, Kobe 657-8501, Japan. ${ }^{7}$ Core Research Division, Organization of Advanced Science and Technology, Kobe University, Kobe 657-8501, Japan. ${ }^{8}$ Institute of Experimental Botany, Centre of the Region Haná for Biotechnological and Agricultural Research, CZ-78371 Olomouc, Czech Republic. ${ }^{9}$ Wheat Breeding Group, NARO Tohoku Agricultural Research Center, Morioka 020-0198, Japan. ${ }^{10}$ Institute of Plant Science and Resources, Okayama University, Kurashiki 710-0046, Japan. ${ }^{11}$ Kihara Institute for Biological Research, Yokohama City University, Yokohama 244-0813, Japan.

\section{Received: 6 November 2014 Accepted: 31 July 2015} Published online: 12 August 2015

\section{References}

1. Kihara H, Nishiyama I. Genomanalyse bei Triticum und Aegilops. I Genomaffinitäten in tri-, tetra- und pentaploiden Weizenbastarden. Cytologia. 1930;1:270-84

2. Sears ER. The aneuploids of common wheat. Missouri Agricultural Experiment Station Research Bulletin. 1954;572:1-58.

3. Okamoto M. Identification of the chromosomes of common wheat belonging to the A and B genome. Can J Genet Cytol. 1962;4:31-7.

4. Šafář J, Šimková H, Kubaláková M, Číhalíková J, Suchánková P, Bartoš J, et al. Development of chromosome-specific BAC resources for genomics of bread wheat. Cytogenet Genome Res. 2010;129:211-23.

5. International Rice Genome Sequencing Project. The map-based sequence of the rice genome. Nature. 2005:436:793-800

6. International Brachypodium Initiative. Genome sequencing and analysis of the model grass Brachypodium distachyon. Nature. 2010:463:763-8.

7. Paterson AH, Bowers JE, Bruggmann R, Dubchak I, Grimwood J, Gundlach H, et al. The Sorghum bicolor genome and the diversification of grasses. Nature. 2009:457:551-6.
8. Schnable PS, Ware D, Fulton RS, Stein JC, Wei F, Pasternak S, et al. The B73 maize genome: complexity, diversity, and dynamics. Science. 2009;326:1112-5

9. The International Barley Genome Sequencing Consortium. A physical, genetic and functional sequence assembly of the barley genome. Nature. 2012:491:711-6.

10. Flavell RB, Rimpau J, Smith DB. Repeated sequence DNA relationships in 4 cereal genomes. Chromosoma. 1977;63:205-22.

11. Brenchley R, Spannagl M, Pfeifer M, Barker GLA, D'Amore R, Allen AM, et al. Analysis of the bread wheat genome using whole-genome shotgun sequencing. Nature. 2012;491:705-10.

12. Ling HQ, Zhao S, Liu D, Wang J, Sun H, Zhang C, et al. Draft genome of the wheat A-genome progenitor Triticum urartu. Nature. 2013;496:87-90.

13. Jia J, Zhao S, Kong X, Li Y, Zhao G, He W, et al. Aegilops tauschii draft genome sequence reveals a gene repertoire for wheat adaptation. Nature. 2013:496:91-5

14. Doležel J, Kubaláková M, Paux E, Bartoš J, Feuillet C. Chromosome-based genomics in the cereals. Chromosome Res. 2007;15:51-66.

15. The International Wheat Genome Sequencing Consortium. A chromosome-based draft sequence of the hexaploid bred wheat genome. Science. 2014;345:1251788.

16. Breen J, Wicker T, Shatalina M, Frenkel Z, Bertin I, Phillippe R, et al. A physical map of the short arm of wheat chromosome 1A. PLoS One. 2013:11:e80272.

17. Lucas SJ, Akpınar BA, Kantar M, Weinstein Z, Aydınoğlu F, Šafár J, et al. Physical mapping integrated with syntenic analysis to characterize the gene space of the long arm of wheat chromosome 1A. PLoS One. 2013;8:e59542.

18. Raats D, Frenkel Z, Krugman T, Dodek I, Sela H, Šimková H, et al. The physical map of wheat chromosome 1BS provides insights into its gene space organization and evolution. Genome Biol. 2013;14:R138.

19. Philippe R, Paux E, Bertin I, Sourdille P, Choulet F, Laugier C, et al. A high density physical map of chromosome 1BL advances evolutionary studies, map-based cloning and sequencing in wheat. Genome Biol. 2013;14:R64.

20. Paux E, Sourdille P, Salse J, Saintenac C, Choulet F, Leroy P, et al. A physical map of the 1-gigabase bread wheat chromosome 3B. Science. 2008;322:101-4.

21. Poursarebani N, Nussbaumer T, Šimková H, Šafár J, Witsenboer H, van Oeveren J, et al. Whole genome profiling (WGP'M ) and shotgun sequencing delivers an anchored, gene decorated, physical map assembly of bread wheat chromosome 6A. Plant J. 2014;79:334-47.

22. Cviková K, Cattonaro F, Alaux M, Stein N, Mayer KFX, Doležel J, et al. High-throughput physical map anchoring via BAC-pool sequencing. BMC Plant Biol. 2015;15:99.

23. Akpinar BA, Magni F, Yuce M, Lucas SJ, Šimková H, Šafár J, et al. The physical map of wheat chromosome 5DS revealed gene duplications and small rearrangements. BMC Genomics. 2015;16:453.

24. Tanaka T, Kobayashi F, Joshi G, Onuki R, Sakai H, Kanamori H, et al. Next-generation survey sequencing and the molecular organization of wheat chromosome 6B. DNA Res. 2014:21:103-14.

25. Pikaard CS. The epigenetics of nucleolar dominance. Trends Genet. 2000:16:495-500.

26. Morrison JW. Chromosome behavior in wheat monosomics. Heredity. 1953;7:203-17.

27. Mclntosh RA, Yamazaki Y, Dubcovsky J, Rogers J, Morris C, Apples R, et al. Catalogue of gene symbols for wheat. 2013 [http://www.shigen.nig.ac.jp/ wheat/komugi/genes/download.jsp]

28. Paux E, Roger D, Badaeva E, Gay G, Bernard M, Sourdille P, et al. Characterizing the composition and evolution of homoeologous genomes in hexaploid wheat through BAC end sequencing on chromosome $3 \mathrm{~B}$. Plant J. 2006:48:463-74.

29. Kalavacharla V, Hossain K, Gu Y, Riera-Lizarazu O, Vales MI, Bhamidimarri S, et al. High-resolution radiation hybrid map of wheat chromosome 1D. Genetics. 2006;173:1089-99.

30. Sears E, Sears L. The telocentric chromosomes of common wheat. In: Proc 5th Int Wheat Genet Symp. New Delhi, India: Agricultural Research Institute; 1978. p. 389-407.

31. van Oeveren J, de Ruiter M, Jesse T, van der Poel H, Tang J, Yalcin F, et al. Sequence-based physical mapping of complex genomes by whole genome profiling. Genome Res. 2011;21:618-25.

32. Philippe $R$, Choulet F, Paux E, van Oeveren J, Tang J, Wittenberg AH, et al. Whole genome profiling provides a robust framework for physical mapping and sequencing in the highly complex and repetitive wheat genome. BMC Genomics. 2012;13:47. 
33. Soderlund C, Longden I, Mott R. FPC: a system for building contigs from restriction fingerprinted clones. Comput Appl Biosci. 1997;13:523-35.

34. Ishikawa G, Yonemaru J, Saito M, Nakamura T. PCR-based landmark unique gene (PLUG) markers effectively assign homoeologous wheat genes to A, B and D genomes. BMC Genomics. 2007;8:135

35. Ishikawa G, Nakamura T, Ashida T, Saito M, Nasuda S, Endo TR, et al. Localization of anchor loci representing five hundred annotated rice genes to wheat chromosomes using PLUG markers. Theor Appl Genet. 2009;118:499-514.

36. Mayer KFX, Martis M, Hedley PE, Šimková H, Liu H, Morris JA, et al. Unlocking the barley genome by chromosomal and comparative genomics. Plant Cell. 2011;23:1249-63.

37. Kobayashi F, Takumi S, Handa H. Identification of quantitative trait loci for $A B A$ responsiveness at the seedling stage associated with $A B A$-regulated gene expression in common wheat. Theor Appl Genet. 2010;121:629-41.

38. Tiwari VK, Riera-Lizarazu O, Gunn HL, Lopez K, Iqbal MJ, Kianian S, et al. Endosperm tolerance of paternal aneuploidy allows radiation hybrid mapping of the wheat D-genome and a measure of $\gamma$ ray-induced chromosome breaks. PLoS One. 2012;7:e48815.

39. Endo TR, Gill BS. The deletion stocks of common wheat. J Hered. 1996:87:295-307.

40. Qi L, Echalier B, Friebe B, Gill BS. Molecular characterization of a set of wheat deletion stocks for use in chromosome bin mapping of ESTs. Funct Integr Genomics. 2003;3:39-55.

41. Sourdille P, Singh S, Cadalen T, Brown-Guedira GL, Gay G, Qi L, et al. Microsatellite-based deletion bin system for the establishment of genetic-physical map relationships in wheat (Triticum aestivum L.). Funct Integr Genomics. 2004;4:12-25.

42. La Rota M, Sorrells ME. Comparative DNA sequence analysis of mapped wheat ESTs reveals the complexity of genome relationships between rice and wheat. Funct Integr Genomics. 2004:4:34-46.

43. Randhawa HS, Dilbirlig M, Sidhu D, Erayman M, Sandhu D, Bondareva S, et al. Deletion mapping of homoeologous group 6-specific wheat expressed sequence tags. Genetics. 2004;168:677-86.

44. Barker RF, Harberd NP, Jarvis MG, Flavell RB. Structure and evolution of the intergenic region in a ribosomal DNA repeat unit of wheat. J Mol Biol. 1988;201:1-17.

45. Flavell RB, O'Dell M. Ribosomal RNA genes on homoeologous chromosomes of group 5 and 6 in hexaploid wheat. Heredity. 1976;37:377-85.

46. Gerlach WL, Bedbrook JR. Cloning and characterization of ribosomal RNA genes from wheat and barley. Nucleic Acids Res. 1979;7:1870-85.

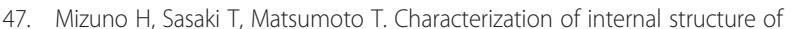
the nucleolar organizing region in rice (Oryza sativa L.). Cytogenet Genome Res. 2008;121:282-5.

48. Kawahara Y, de la Bastide M, Hamilton JP, Kanamori H, McCombie WR, Ouyang S, et al. Improvement of the Oryza sativa Nipponbare reference genome using next generation sequence and optical map data. Rice. 2013;6:4.

49. Fujisawa M, Yamagata H, Kamiya K, Nakamura M, Saji S, Kanamori H, et al. Sequence comparison of distal and proximal ribosomal DNA arrays in rice (Oryza sativa L.) chromosome $9 S$ and analysis of their flanking regions. Theor Appl Genet. 2006;113:419-28.

50. Komuro S, Endo R, Shikata K, Kato A. Genomic and chromosomal distribution patterns of various repeated DNA sequences in wheat revealed by a fluorescence in situ hybridization procedure. Genome. 2013;56:131-7.

51. Li B, Choulet F, Heng Y, Hao W, Paux E, Liu Z, et al. Wheat centromeric retrotransposons: the new ones take a major role in centromeric structure. Plant J. 2013;73:952-65.

52. Fukui KN, Suzuki G, Kagudah ES, Rahman S, Apples R, Yamamoto M, et al. Physical arrangement of retrotransposon-related repeats in centromeric regions of wheat. Plant Cell Physiol. 2001;42:189-96.

53. Liu Z, Yue W, Li D, Wang RR, Kong X, Lu K, et al. Structure and dynamics of retrotransposons at wheat centromeres and pericentromeres. Chromosoma. 2008;10:445-56.

54. Qi L, Friebe B, Zhang P, Gill BS. A molecular-cytogenetic method for locating genes to pericentromeric regions facilitates a genomewide comparison of synteny between the centromeric regions of wheat and rice. Genetics. 2009;183:1235-47.

55. Rustenholz C, Choulet F, Laugier C, Šafár J, Šimková H, Doležel J, et al. A 3,000-Loci transcription map of chromosome 3B unravels the structural and functional features of gene islands in hexaploid wheat. Plant Physiol. 2011;157:1596-608.

56. Choulet F, Alberti A, Theil S, Glover N, Barbe V, Daron J, et al. Structural and functional partitioning of bread wheat chromosome 3B. Science. 2014;345:1249721-1.

57. Gale MD, Devos KM. Comparative genetics in the grasses. Proc Natl Acad Sci U S A. 1998;95:1971-4.

58. Devos KM. Updating the 'Crop Circle'. Curr Opin Plant Biol. 2005;8:155-62.

59. Abrouk M, Murat F, Pont C, Messing J, Jackson S, Faraut T, et al. Palaeogenomics of plants: synteny-based modeling of extinct ancestors. Trends Plant Sci. 2010;15:479-87.

60. Luo M-C, Gu YQ, You FM, Deal KR, Ma Y, Hu Y, et al. A 4-gigabase physical map unlock the structure and evolution of the complex genome of Aegilops tauschii, the wheat D-genome progenitor. Proc Natl Acad Sci U S A. 2013:110:7940-5

61. Vrána J, Kubaláková M, Šimková H, Číhalíková J, Lysák MA, Doležel J. Flow-sorting of mitotic chromosomes in common wheat (Triticum aestivum L.). Genetics. 2000;156:2033-41.

62. Janda J, Šafář J, Kubaláková M, Bartoš J, Kovářová P, Suchánková P, et al. Advanced resources for plant genomics: BAC library specific for the short arm of wheat chromosome 1B. Plant J. 2006;47:977-86.

63. Šimková H, Šafář J, Kubaláková M, Suchánková P, Číhalíková J, Robert-Quatre H, et al. BAC libraries from wheat chromosome 7D: efficient tool for positional cloning of aphid resistance genes. J Biomed Biotechnol. 2011;2011:302543.

64. Nelson W, Soderlund C. Integrating sequence with FPC fingerprint maps. Nucleic Acids Res. 2009:37:e36.

65. Altschul SF, Madden TL, Schaffer AA, Zhang JH, Zhang Z, Miller W, et al. Gapped BLAST and PSI-BLAST: a new generation of protein database search programs. Nucleic Acids Res. 1997;25:3389-402.

66. Mochida K, Yoshida T, Sakurai T, Ogihara Y, Shinozaki K. TriFLDB: a database of clustered full-length coding sequences from Triticeae with applications to comparative grass genomics. Plant Physiol. 2009;150:1135-46.

67. Matsumoto T, Tanaka T, Sakai H, Amano N, Kanamori H, Kurita K, et al. Comprehensive sequence analysis of 24,783 barley full-length cDNAs derived from 12 clone libraries. Plant Physiol. 2011:156:20-8.

68. Birnboim HC, Doly J. A rapid alkaline extraction procedure for screening recombinant plasmid DNA. Nucleic Acids Res. 1979:7:1513-23.

69. de Givry S, Bouchez M, Chabrier P, Milan D, Schiex T. Carthagene: multipopulation integrated genetic and radiation hybrid mapping. Bioinformatics. 2005;21:1703-4

70. Huang X, Madan A. CAP3: A DNA Sequence Assembly Program. Genome Res. 1999:9:868-77.

71. Krzywinski M, Schein J, Birol I, Connors J, Gascoyne R, Horsman D, et al. Circos: An information aesthetic for comparative genomics. Genome Res. 2009;19:1639-45

72. Armachea J-P, Jarascha A, Angera AM, Villab E, Beckera T, Bhushana S, et al. Cryo-EM structure and rRNA model of a translating eukaryotic $80 \mathrm{~S}$ ribosome at 5.5-Å resolution. Proc Natl Acad Sci U S A. 2010:107:19748-53.

\section{Submit your next manuscript to BioMed Central and take full advantage of:}

- Convenient online submission

- Thorough peer review

- No space constraints or color figure charges

- Immediate publication on acceptance

- Inclusion in PubMed, CAS, Scopus and Google Scholar

- Research which is freely available for redistribution 